hep-th/0207105

\title{
Time Evolution in Open String Theory
}

\author{
Ashoke Sen \\ Harish-Chandra Research Institute \\ Chhatnag Road, Jhusi, Allahabad 211019, INDIA \\ and \\ Department of Physics, Penn State University \\ University Park, PA 16802, USA \\ E-mail: asen@thwgs.cern.ch, sen@mri.ernet.in
}

\begin{abstract}
We discuss a general iterative procedure for constructing time dependent solutions in open string theory describing rolling of a generic tachyon field away from its maximum. These solutions are characterized by two parameters labelling the initial position and velocity of the tachyon field, but one of these parameters can be removed by using time translation symmetry. The Wick rotated version of the resulting one parameter family of inequivalent solutions describes a one parameter family of boundary conformal field theories, each member of which is related to the boundary conformal field theory describing the original D-brane system by a nearly marginal deformation. We apply this technique to construct a time dependent solution on a D-brane in bosonic string theory which can be interpreted as the creation of a lower dimensional brane during the decay of an unstable D-brane.
\end{abstract}




\section{Contents}

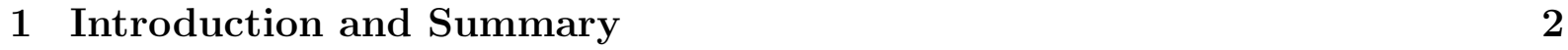

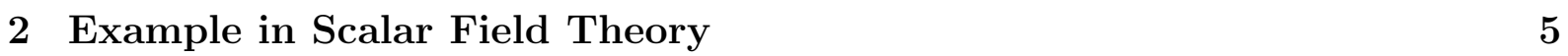

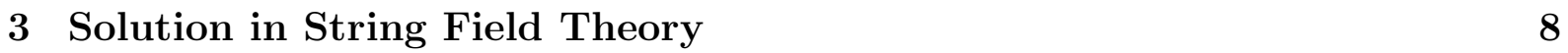

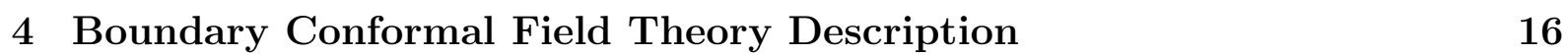

$\begin{array}{lll}5 & \text { Explicit Example } & 19\end{array}$

6 Time Dependent Solutions on D-branes in Superstring Theories 25

\begin{tabular}{|lll}
\hline & Tachyon Dynamics in Closed String Theory & 29
\end{tabular}

\begin{tabular}{lll}
\hline & Conclusion & 30
\end{tabular}

\section{Introduction and Summary}

Time dependent solutions in string theory have received attention recently, both in closed string theory [1] and in open string theory[2, 3, [, 5, 6, 7, 8, 9]. (For early studies of open string tachyons see [10].) In particular in open string theory a special class of time dependent solutions were constructed which describe the rolling of the tachyon field on a non-BPS D-brane[11, 12], or a brane-antibrane pair away from the maximum of the potential. Possible applications of these solutions to cosmology have been discussed in [13. .1

One common feature of the solutions associated with the rolling of an open string tachyon is that after removing the trivial parameter of the solution associated with time translation, the solutions are characterized by one parameter labelling the energy of the solution. This is precisely what one would expect in a scalar field theory with standard kinetic term, where a general spatially homogeneous solution will be characterized by the initial position and velocity of the scalar field, and one of these two parameters can be removed by using time translation invariance which allows us to set either the initial position or the initial velocity to zero.t However this is somewhat surprising in open

\footnotetext{
${ }^{1}$ For earlier attempts to apply the rolling tachyon solution to cosmology, see [14].

${ }^{2}$ In a multi-well potential there can be more than one family of inequivalent solutions labelled by the energy.
} 
string field theory where the interaction terms have infinite number of derivatives.'f

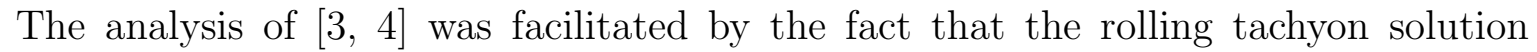
was related by Wick rotation to a boundary conformal field theory (BCFT) that was obtained from the original D-brane system by an exactly marginal deformation. The deformation parameter was related to the parameter labelling the inequivalent solutions. We cannot hope that this will be true for more general tachyonic states which might be present on a generic D-brane system, e.g. tachyons on an intersecting brane system 15, 16. Thus the question arises: is it still possible to construct a one parameter family of inequivalent solutions which describe the rolling of a generic open string tachyon away from its maximum?

This is the question we address in the paper, and show that under certain generic conditions, the answer to this question is in the affirmative. In particular once these conditions are satisfied, we have a one parameter family of inequivalent solutions, which are related by Wick rotation to a one parameter family of euclidean BCFT's. However, these BCFT's are not related to each other by a a marginal deformation. Instead, each of them is related to the BCFT describing the original unstable D-brane system by a nearly marginal deformation. The nearly marginal operator, however, is different for different solutions.

Unfortunately, although our analysis establishes the existence of a one parameter family of rolling tachyon solutions for a generic open string tachyon, unlike in the cases discussed in [3, 4], generically the deformed BCFT's are not exactly solvable. As a result, we cannot give an explicit construction of the energy-momentum tensor by Wick rotating the boundary state of the deformed theory. Nevertheless, the success of this approach in generating explicit solution in special cases where the deformation is exactly marginal [3, 4, as well as in generating general class of solutions in $p$-adic string theory [18] leads us to believe that this approach has a more general validity.

In section 2 we illustrate our method of constructing the rolling tachyon solution by studying the example of a scalar field theory with standard kinetic term and potential $V(\phi)$ with a maximum at $\phi=0$. The idea is to relate the rolling tachyon solution via Wick rotation to the equation of motion for a scalar field with potential $-V(\phi)$. We can now solve for the periodic motion of this scalar field around $\phi=0$ using perturbation theory where we let the period of oscillation depend on the amplitude, and solve for the period and the orbit as a perturbation series in the amplitude 19]. Once we have

\footnotetext{
${ }^{3}$ Of course while the analysis of [3, 4 , generates a one parameter family of time dependent solutions, it does not rule out the existence of other solutions and so it is still conceivable that the full theory has more solutions.

${ }^{4}$ Possible application of rolling tachyon on intersecting D-brane system to cosmology has been emphasized in 17.
} 
obtained a solution this way we can inverse Wick rotate the solution to find a rolling tachyon solution. The amplitude of oscillation in the Wick rotated theory labels the initial value of the rolling tachyon.

In section 3 we show how under certain conditions this procedure can be generalized to open string field theory to construct rolling tachyon solutions associated with a generic tachyon field. We also generalize this construction to the case where multiple tachyons roll simultaneously beginning with arbitrary initial position and velocity. We reinterpret this construction in section $⿴$ ) in terms of boundary conformal field theory. In particular we show that in the Wick rotated theory, the family of solutions labelled by the initial position of the tachyon field correspond to a family of BCFT's, and each member of this family is related to the BCFT describing the original D-brane by a nearly marginal deformation.

In section 5 we consider a specific example - that of a D-p-brane in bosonic string theory with one direction compactified on a circle of radius $R$, - and consider the rolling of the $(p-1,1)$ dimensional tachyon, obtained by taking the first momentum mode of the $(p, 1)$ dimensional tachyon along the circle. We show how in the Wick rotated theory we can construct a one parameter family of boundary conformal field theories, each related to the original D-p-brane by a nearly marginal deformation, and how the inverse Wick rotation of these BCFT's generate the family of rolling tachyon solutions. Unfortunately these BCFT's are not exactly solvable and hence we cannot compute the analytic expressions for the energy-momentum tensor associated with these solutions. However at a particular value of the radius, $R=\sqrt{2}$, the family of BCFT's become related by a marginal deformation and are exactly solvable. As a result we can compute the time evolution of the energy momentum tensor explicitly by taking the inverse Wick rotation of the boundary states associated with these BCFT's. We find that during the course of time evolution there is non-trivial flow of energy density along the compact direction, and at a certain finite value of time, the energy density at a particular location on this circle blows up. More specifically, the energy density as a function of the coordinate $y$ along the compact circle approaches a delta function singularity as we approach this critical time. As we pass this critical time, there is an apparent loss of energy density at the location of the singularity, with the total amount of energy lost being equal to the total initial energy of the system. Thus just after the critical time the energy density averages to zero. If we naively continue the formula for energy density beyond this critical time, the energy density gradually evolves to zero everywhere. We suggest a natural interpretation of this as the creation of a codimension one lump from the decay of the original brane.

In section 6 we generalize the construction to the case of superstring theory. In particular we consider rolling tachyon solution that should describe the creation of a $\mathrm{D}-(p-1)$ 
- $\overline{\mathrm{D}}-(p-1)$ pair due to the decay of a non-BPS D-p-brane wrapped on a circle. As in the case of bosonic string theory, we can construct this solution in the Wick rotated theory as a nearly marginal deformation of the original D- $p$-brane conformal field theory. However, this BCFT is not exactly solvable, and hence we cannot explicitly construct the space-time dependent energy momentum tensor associated with this solution. In section 7 we discuss possible generalization of this method to the study of closed string tachyon condensation. Although part of the argument can be generalized to the case of closed strings, the main obstacle to finding the solution in closed string theory arises due to our inability to solve the equations of motion of the graviton and the dilaton field in the background of rolling tachyon. We conclude in section 8 with a few remarks.

\section{Example in Scalar Field Theory}

In this section we shall discuss the general method for constructing time dependent solutions in the context of a scalar field theory, which we shall generalize to string field theory in the later sections. We begin with the action of a scalar field $\phi$ in $p+1$ dimensions with standard kinetic term and potential $V(\phi)$ :

$$
S=-\int d^{p+1} x\left[\partial^{\mu} \phi \partial_{\mu} \phi+V(\phi)\right] .
$$

We shall further assume that $V(\phi)$ has a maximum at $\phi=0$, with

$$
V^{\prime \prime}(0)=-m^{2} .
$$

We want to study time dependent solution of this equation determined by a given initial condition. For simplicity let us consider spatially homogeneous field configurations. In this case the solution is characterized by two parameters, - the initial position and velocity of $\phi$. For definiteness we shall further restrict to solution with total energy $E<V(0)$. In this case $\partial_{0} \phi$ vanishes at some instant of time when $V(\phi)=E$. We shall take this to be the origin of $x^{0}$. Thus the solution is now characterized by only one parameter $\lambda$, the value of $\phi$ at $x^{0}=0$. We shall be interested in the solution where $\lambda$ is small but not infinitesimal.

The equation of motion is:

$$
\partial_{0}^{2} \phi+V^{\prime}(\phi)=0 .
$$

It is of course straightforward to (numerically) integrate this equation, but this cannot be generilized to string field theory where the equations of motion contain infinite number of derivatives. We shall follow an indirect method that can be generalized in the context 
of string field theory. The basic idea is the same as the one followed in [3], 4], namely we make a Wick rotation $x^{0}=i x$, and write eq.(2.3) as

$$
\partial_{x}^{2} \phi-V^{\prime}(\phi)=0
$$

If $\phi=f(x)$ is a solution of eq.(2.4), then $\phi=f\left(-i x^{0}\right)$ will be a solution to (2.3). Thus the aim is now to solve eq.(2.4) with the boundary condition $\phi=\lambda, \partial_{x} \phi=0$ at $x=0$. This clearly can be thought of as a motion of a particle in potential $-V(\phi)$. Since $V(\phi)$ has a maximum at $\phi=0,-V(\phi)$ has a minimum at $\phi=0$, and for small $\lambda$, solution to the equation of motion will oscillate around zero. The period of oscillaton $T \equiv 2 \pi / \omega$ is in general a function of $\lambda$. Thus the solution can be expanded as

$$
\phi(x ; \lambda)=\sum_{n=0}^{\infty} a_{n} \cos (n \omega x) .
$$

We shall now solve (2.4) using perturbation theory [19]. If we write

$$
V(\phi)=-\frac{1}{2} m^{2} \phi^{2}+V_{\text {int }}(\phi)
$$

then (2.4) takes the form

$$
\left(\partial_{x}^{2}+m^{2}\right) \phi-V_{\text {int }}^{\prime}(\phi)=0 .
$$

$V_{\text {int }}(\phi)$ is of order $\phi^{n}$ with $n \geq 3$. Thus for small $\lambda$ the solution behaves as

$$
\phi=\lambda \cos (m x)+\mathcal{O}\left(\lambda^{2}\right)
$$

Comparing this with (2.5) we get

$$
a_{1}=\lambda+\mathcal{O}\left(\lambda^{2}\right), \quad \omega=m+\mathcal{O}(\lambda), \quad a_{n}=\mathcal{O}\left(\lambda^{2}\right) \quad \text { for } \quad n=0, n \geq 2 .
$$

From this we see that we can trade in the parameter $\lambda$ for the coefficient $a_{1} \equiv \hat{\lambda}$, and rewrite (2.9) as

$$
a_{1}=\widehat{\lambda}, \quad \omega=m+\mathcal{O}(\widehat{\lambda}), \quad a_{n}=\mathcal{O}\left(\widehat{\lambda}^{2}\right) \quad \text { for } \quad n=0, n \geq 2 .
$$

${ }^{5} \mathrm{An}$ analytic expression of $T$ is given by

$$
T=\sqrt{2} \int_{\phi_{1}}^{\phi_{2}} \frac{d \phi}{\sqrt{V(\phi)-V(\lambda)}}
$$

where $\phi_{1}$ and $\phi_{2}$ are turning points where $V(\lambda)=V\left(\phi_{1}\right)=V\left(\phi_{2}\right)$. The initial value $\lambda$ of $\phi$ can be identified with either $\phi_{1}$ or $\phi_{2}$. However, we shall not use this formula. 
We now substitute (2.5) into eq.(2.7) to get

$$
\sum_{n=0}^{\infty}\left(n^{2} \omega^{2}-m^{2}\right) a_{n} \cos (n \omega x)=-V_{i n t}^{\prime}\left(\sum_{n=0}^{\infty} a_{n} \cos (n \omega x)\right) .
$$

The right hand side of this equation contains terms quadratic and higher order in $a_{n}$. Thus we can solve the equations iteratively as follows. The zeroeth order approximation is taken to be

$$
\omega=m, \quad a_{n}=0 \quad \text { for } \quad n=0, \quad n \geq 2 .
$$

Also $a_{1}$ is set equal to $\hat{\lambda}$ to all orders. We substitute the $k$-th order results for the $a_{n}$ 's and $\omega$ on the right hand side of (2.11) to compute the coefficient of $\cos (n \omega x)$ for every $n$. Comparing this with the coefficient of $\cos (n \omega x)$ on the left hand side, we determine the $(k+1)$ th order values of the $a_{n}$ 's for $n=0$ and $n \geq 2$. On the other hand equating the coefficient of the $\cos (\omega x)$ terms on both sides, we determine the value of $\omega$ to $(k+1)$ th order. This is possible since $a_{1}$ is set equal to $\hat{\lambda}$, and we are determining all the coefficients in terms of $\hat{\lambda}$.

This gives a solution to eq.(2.4). Given this, we can now generate a solution to eq.(2.3) by making the substitution $x=-i x^{0}$. This gives

$$
\phi\left(x^{0}\right)=\sum_{n=0}^{\infty} a_{n}(\widehat{\lambda}) \cosh \left(n \omega(\widehat{\lambda}) x^{0}\right)
$$

where the coefficients $a_{n}$ are the same as the ones determined by the previous method. This gives a family of solutions characterized by one parameter $\hat{\lambda} . \hat{\lambda}$ determines the initial value $\lambda$ of $\phi$, with the precise relation between $\hat{\lambda}$ and $\lambda$ being given by:

$$
\lambda=\phi(0)=\sum_{n=0}^{\infty} a_{n}(\widehat{\lambda}) .
$$

For example, if we take the $\phi^{3}$ field theory with potential $-\frac{1}{2} \phi^{2}+\frac{1}{3} \phi^{3}$, then $V_{\text {int }}^{\prime}(\phi)=\phi^{2}$. Using the first order solution $\phi\left(x^{0}\right)=\widehat{\lambda} \cos \left(\omega x^{0}\right)$ on the right hand side of (2.11), we get the solution to second order in $\hat{\lambda}$ :

$$
a_{1}=\widehat{\lambda}, \quad a_{0} \simeq \frac{1}{2} \widehat{\lambda}^{2}, \quad a_{2} \simeq-\frac{1}{6} \widehat{\lambda}^{2}, \quad \omega \simeq 1
$$

Note that $\omega$ remains constant at 1 since the Fourier expansion of $(\widehat{\lambda} \cos x)^{2}$ does not contain a term proportional to $\cos x$. Substituting (2.15) on the right hand side of (2.11)

\footnotetext{
${ }^{6}$ Actually this determines $\omega$ to order $\widehat{\lambda}^{k}$, but since the coefficients $a_{n}$ are of order $\widehat{\lambda}$ or higher, this determines the solution to order $\widehat{\lambda}^{k+1}$.
} 


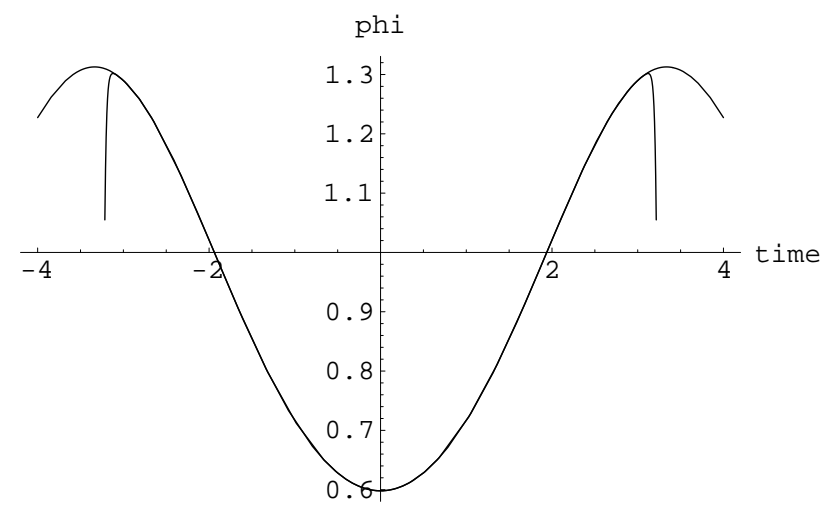

Figure 1: The plot of a particular time dependent solution in a scalar field theory with potential $-\phi^{2} / 2+\phi^{3} / 3$ for the choice $\widehat{\lambda}=.5$. This gives $\phi\left(x^{0}=0\right) \simeq 0.598122$. The top curve is the result of direct numerical integration with this boundary condition, while the bottom curve is obtained by perturbative techniques discussed here with 60 harmonics. As we can see the two curves coincide for $-\tau / 2<x<\tau / 2$ where $\tau$ is the period of oscillation, but the perturbation theory breaks down beyond this range.

and comparing coefficients of $\cos (\omega x)$ on both sides we get the equation for $\omega$ at next order:

$$
\left(\omega^{2}-1\right) \hat{\lambda} \simeq-\frac{5}{6} \widehat{\lambda}^{3} \quad \rightarrow \quad \omega^{2} \simeq 1-\frac{5}{6} \widehat{\lambda}^{2}
$$

The coefficients $a_{n}$ to order $\widehat{\lambda}^{3}$ are given by:

$$
a_{1}=\widehat{\lambda}, \quad a_{0} \simeq \frac{1}{2} \widehat{\lambda}^{2}, \quad a_{2} \simeq-\frac{1}{6} \widehat{\lambda}^{2}, \quad a_{3} \simeq \frac{1}{48} \widehat{\lambda}^{3} .
$$

The explicit expression for the solution becomes complicated at higher order, but we can evaluate it numerically. As illustrated in Fig.1, explicit numerical analysis in this $\phi^{3}$ field theory shows that this procedure generates the time dependent solution in this theory, describing rolling of $\phi$ from the maximum towards the local minimum of $V(\phi)$, quite accurately for half a period of oscillation in either direction of $x^{0}$. Beyond this range the expansion (2.13) diverges and we must take recourse to analytic continuation in order to study the solution.

\section{Solution in String Field Theory}

We now consider some specific D-brane system in bosonic string theory with a tachyonic mode. Associated with the zero momentum tachyon state, there is a boundary operator 
$V_{T}$ of dimension $h<1$. In $\alpha^{\prime}=1$ unit, the associated tachyon field has mass ${ }^{2}$

$$
(h-1) \equiv-m^{2} .
$$

If we displace the tachyon field $T$ a distance $\lambda$ away from its maximum, and let the system evolve in time, then to leading order in $\lambda$ the solution is given by $\lambda \cosh \left(m x^{0}\right)$. In the Wick rotated theory this corresponds to $\lambda \cos (m x)$. The corresponding solution of the linearizerd equations of motion in string field theory is given by

$$
\lambda \cos (m X(0)) V_{T}(0) c_{1}|0\rangle .
$$

This is a BRST invariant state of $L_{0}$ eigenvalue 0 .

The full string field theory equation of motion is given by:

$$
Q_{B}|\Psi\rangle+|\Psi * \Psi\rangle=0
$$

where $|\Psi\rangle$ is an open string state of ghost number 1 , and $Q_{B}$ is the BRST charge. We shall try to find a solution to eq.(3.3) in the Siegel gauge

$$
b_{0}|\Psi\rangle=0,
$$

where the equation takes the form:

$$
L_{0}|\Psi\rangle=-b_{0}|\Psi * \Psi\rangle .
$$

Our goal will be to first construct a solution to (3.5) that extends (3.2) to higher orders in $\lambda$, and then carry out an inverse Wick rotation to construct a time dependent solution to eq.(3.5) describing the rolling of the tachyon away from its maximum. We begin by defining:

$$
\left|\phi_{0}\right\rangle=\cos (\omega X(0)) V_{T}(0) c_{1}|0\rangle,
$$

where $\omega$ is a number that will be determined below. We choose a set of linearly independent basis states $\left|\phi_{r}\right\rangle(0 \leq r<\infty)$ in a subspace $\mathcal{H}_{1}$ of first quantized open string states, satisfying the following conditions:

1. Each $\left|\phi_{r}\right\rangle$ has ghost number 1 .

2. Each $\left|\phi_{r}\right\rangle$ is symmetric under $X \rightarrow-X$, and carries $x$-momentum which is an integer multiple of $\omega$.

3. All $\left|\phi_{r}\right\rangle$ for $r \geq 1$ are orthogonal to $c_{0}\left|\phi_{0}\right\rangle$. 
$\mathcal{H}_{1}$ by definition is the subspace spanned by the basis states $\left\{\left|\phi_{r}\right\rangle, r \geq 0\right\}$ satisfying these properties, and we shall look for a solution $|\Psi\rangle$ of eq.(3.5) in this subspace. We shall also require that when we express the solution $|\Psi\rangle$ as a linear combination of the basis states $\left|\phi_{r}\right\rangle$, the coefficient of $\left|\phi_{0}\right\rangle$ in $|\Psi\rangle$ is given by $\hat{\lambda}$; this in fact will be the definition of the parameter $\hat{\lambda}$. For later use, we shall define:

$$
|\chi\rangle^{\prime}=|\chi\rangle-\mathcal{N}^{-1}\left\langle\phi_{0} \mid \chi\right\rangle c_{0}\left|\phi_{0}\right\rangle, \quad \mathcal{N} \equiv\left\langle\phi_{0}\left|c_{0}\right| \phi_{0}\right\rangle
$$

for any state of $|\chi\rangle$ ghost number 2. Physically $|\chi\rangle^{\prime}$ gives $|\chi\rangle$ with its component along $c_{0}\left|\phi_{0}\right\rangle$ removed.

The construction of the solution $\left|\Psi_{(n)}\right\rangle$ to order $\hat{\lambda}^{n}$ proceeds as follows. We define

$$
\left|\Psi_{1}\right\rangle=\hat{\lambda}\left|\phi_{0}\right\rangle
$$

and $\left|\Psi_{p}\right\rangle$ iteratively by using the relation:

$$
\left|\Psi_{p}\right\rangle=-\frac{b_{0}}{L_{0}}\left(\left|\Psi_{p-1} * \Psi_{p-1}\right\rangle\right)^{\prime}+\widehat{\lambda}\left|\phi_{0}\right\rangle
$$

We repeat (3.9) $(n-1)$-times to compute $\left|\Psi_{n}\right\rangle$. If we define

$$
f_{n}(\omega, \hat{\lambda})=\mathcal{N}^{-1}\left\langle\phi_{0} \mid \Psi_{n-1} * \Psi_{n-1}\right\rangle
$$

then we have

$$
\left(\left|\Psi_{n-1} * \Psi_{n-1}\right\rangle\right)^{\prime}=\left|\Psi_{n-1} * \Psi_{n-1}\right\rangle-f_{n}(\omega, \widehat{\lambda}) c_{0}\left|\phi_{0}\right\rangle .
$$

We now define $\omega_{n}$ as the solution of the equation:

$$
\widehat{\lambda}\left(\omega_{n}^{2}-m^{2}\right)+f_{n}\left(\omega_{n}, \widehat{\lambda}\right)=0,
$$

and take the $n$-th order solution $\left|\Psi_{(n)}\right\rangle$ to be

$$
\left|\Psi_{(n)}\right\rangle=\left|\Psi_{n}\right\rangle_{\omega=\omega_{n}}
$$

Note that $\omega$ at $n$th order is determined after we have computed $f_{n}(\omega, \hat{\lambda})$ by repeating the iterative procedure $(n-1)$ times. Since $f_{n}(\omega, \widehat{\lambda})$ is of order $\hat{\lambda}^{2},(3.12)$ gives $\omega_{n}=m+\mathcal{O}(\widehat{\lambda}) . \square$

We shall now show that $\left|\Psi_{(n)}\right\rangle$ determined this way satisfies:

$$
L_{0}\left|\Psi_{(n)}\right\rangle=-b_{0}\left|\Psi_{(n)} * \Psi_{(n)}\right\rangle+\mathcal{O}\left(\widehat{\lambda}^{n+1}\right),
$$

${ }^{7}$ Actually from the definition of $\phi_{0}$ and conservation of $X$-momentum, it follows that $\left|\phi_{0} * \phi_{0}\right\rangle$ contains states with $X$-momentum $\pm 2 \omega$ or 0 , but does not contain a state of momentum $\pm \omega$. Thus $\left|\phi_{0} * \phi_{0}\right\rangle$ does not contain any component along $c_{0}\left|\phi_{0}\right\rangle$. As a result the order $\widehat{\lambda}^{2}$ contribution to $f_{n}(\omega, \widehat{\lambda})$ vanishes, and $f_{n}(\omega, \widehat{\lambda})=\mathcal{O}\left(\widehat{\lambda}^{3}\right)$. Thus $\omega=m+\mathcal{O}\left(\widehat{\lambda}^{2}\right)$. Generalising this argument we can see that $f_{n}(\omega, \widehat{\lambda})$ must be an odd function of $\widehat{\lambda}$, and hence $\omega$ must be an even function of $\widehat{\lambda}$. 
and hence satisfies the string field theory equations of motion to order $\hat{\lambda}^{n}$. To do this, we first note that (3.9), (3.11) for $p=n$ gives

$$
L_{0}\left|\Psi_{n}\right\rangle=-b_{0}\left|\Psi_{n-1} * \Psi_{n-1}\right\rangle+f_{n}(\omega, \hat{\lambda})\left|\phi_{0}\right\rangle+\widehat{\lambda}\left(\omega^{2}-m^{2}\right)\left|\phi_{0}\right\rangle,
$$

where we have used the fact that

$$
L_{0}\left|\phi_{0}\right\rangle=\left(\omega^{2}-m^{2}\right)\left|\phi_{0}\right\rangle
$$

Eq.(3.12), (3.15) now give

$$
\left[L_{0}\left|\Psi_{n}\right\rangle+b_{0}\left|\Psi_{n-1} * \Psi_{n-1}\right\rangle\right]_{\omega=\omega_{n}}=0
$$

We shall now show that

$$
\left|\Psi_{p}\right\rangle=\left|\Psi_{p-1}\right\rangle+\mathcal{O}\left(\widehat{\lambda}^{p}\right)
$$

for any $p \leq n$. In that case if we replace $\left|\Psi_{n-1}\right\rangle$ on the right hand side of (3.17) by $\left|\Psi_{n}\right\rangle$, the net error will be of order $\hat{\lambda}^{n+1}$, since $\Psi_{n}$ itself is order $\hat{\lambda}$. This, together with (3.13), would establish (3.14).

In order to establish (3.18) we use proof by induction. We use (3.9) to get:

$$
\left|\Psi_{p+1}\right\rangle-\left|\Psi_{p}\right\rangle=-\frac{b_{0}}{L_{0}}\left(\left|\Psi_{p} * \Psi_{p}\right\rangle-\left|\Psi_{p-1} * \Psi_{p-1}\right\rangle\right)^{\prime} .
$$

Now suppose that (3.18) holds for some value of $p$. In that case the right hand side of (3.19) is of order $\hat{\lambda}^{p+1}$, since $\left|\Psi_{p-1}\right\rangle$ itself is of order $\hat{\lambda}$. Since (3.18) holds for $p=1$, this proves (3.18) for all $p \leq n$.

Note however that for the above argument to go through, we need to ensure that $b_{0}\left(\left|\Psi_{p} * \Psi_{p}\right\rangle-\left|\Psi_{p-1} * \Psi_{p-1}\right\rangle\right)^{\prime}$ does not have a state with $L_{0}$ eigenvalue of order $\hat{\lambda}$, since this will give an additional power of $\hat{\lambda}$ in the denominator from the operation of $1 / L_{0}$ and will destroy the counting of powers of $\widehat{\lambda}$ that we have used. It is precisely due to this reason that we have removed the component of $\left|\Psi_{p} * \Psi_{p}\right\rangle$ along $c_{0}\left|\phi_{0}\right\rangle$ in this expression, since $c_{0}\left|\phi_{0}\right\rangle$ has $L_{0}$ eigenvalue $\left(\omega^{2}-m^{2}\right) \sim \hat{\lambda}$. For our analysis to hold it is important that besides $\left|\phi_{0}\right\rangle$, there are no other states appearing in the computation of $\left|\Psi_{p} * \Psi_{p}\right\rangle$, which has $L_{0}$ eigenvalue of order $\hat{\lambda}$ or less.

This requirement can be reformulated purely in terms of matter sector vertex operators as follows. The possible problematic states appearing in the expression for $\left|\Psi_{p} * \Psi_{p}\right\rangle$ are ghost number 2 states which are not annihilated by $b_{0}$, and has $L_{0}$ eigenvalue 0 in the $\widehat{\lambda} \rightarrow 0$ limit. Such states are of the form:

$$
O(0) c_{0} c_{1}|0\rangle
$$


where $O$ is a matter sector vertex operator whose conformal weight approaches 1 as $\widehat{\lambda} \rightarrow 0$. As long as the theory does not contain any operator of this type which can appear in the operator product of $\cos (\omega X) V_{T}$ with itself (other than $\cos (\omega X) V_{T}$ itself, whose effect has already been taken into account) the analysis given above remains valid. Matter operators of the form $\partial X$ of dimension 1, which could cause potential problems, do not do so since the $X \rightarrow-X$ symmetry prevents their appearance in the operator product of $\cos (\omega X) V_{T}$ with itself.

The situation simplifies if not even $c_{0}\left|\phi_{0}\right\rangle$ appears in the computation of $\left|\Psi_{p} * \Psi_{p}\right\rangle$ for $\omega=m$. In this case we see from $(3.10)$ that $f_{n}(m, \hat{\lambda})$ vanishes identically for arbitrary $\hat{\lambda}$. Thus eq.(3.12) can be solved by setting $\omega=m$. The cases analysed in refs. [3, 1 are of this type.

Once we have obtained the solution in the Wick rotated theory, we can perform an inverse Wick rotation $x=-i x^{0}$ to obtain a time dependent solution in the Lorentzian theory. This amounts to replacing $\cos (\omega X)$ by $\cosh \left(\omega X^{0}\right)$, and the oscillator $\alpha_{m}$ of $X$ by $-i \alpha_{m}^{0}$ of $-i X^{0}$. Performing these operations on $\Psi_{(n)}$ we can generate a time dependent solution of the equations of motion to order $\hat{\lambda}^{n}$. The constant $\hat{\lambda}$ labelling the solution parametrizes the initial condition on the tachyon field. Of course, we expect the series expansion to be valid only for a limited range of $x^{0}$, and for $x^{0}$ beyond this range we need to obtain the result via analytic continuation.

Since the discussion so far has been somewhat abstract, we shall now give explicit form of the solution to order $\hat{\lambda}^{3}$. We have:

$$
\begin{gathered}
\left|\Psi_{1}\right\rangle=\widehat{\lambda}\left|\phi_{0}\right\rangle, \\
\left|\Psi_{2}\right\rangle=\widehat{\lambda}\left|\phi_{0}\right\rangle-\widehat{\lambda}^{2} \frac{b_{0}}{L_{0}}\left|\phi_{0} * \phi_{0}\right\rangle .
\end{gathered}
$$

No subtraction is needed to this order since $\left|\phi_{0} * \phi_{0}\right\rangle$ does not have a component along $c_{0}\left|\phi_{0}\right\rangle$ due to $X$-momentum conservation. To next order 8

$$
\begin{aligned}
\left|\Psi_{3}\right\rangle= & \hat{\lambda}\left|\phi_{0}\right\rangle-\widehat{\lambda}^{2} \frac{b_{0}}{L_{0}}\left|\phi_{0} * \phi_{0}\right\rangle \\
& +\frac{b_{0}}{L_{0}}\left(\widehat{\lambda}^{3}\left|\phi_{0}\right\rangle * \frac{b_{0}}{L_{0}}\left|\phi_{0} * \phi_{0}\right\rangle+\widehat{\lambda}^{3} \frac{b_{0}}{L_{0}}\left|\phi_{0} * \phi_{0}\right\rangle *\left|\phi_{0}\right\rangle+f_{3}(\omega, \widehat{\lambda}) c_{0}\left|\phi_{0}\right\rangle\right) \\
& +\mathcal{O}\left(\widehat{\lambda}^{4}\right)
\end{aligned}
$$

${ }^{8}$ We could ignore the $\mathcal{O}\left(\widehat{\lambda}^{4}\right)$ term in $\left|\Psi_{3}\right\rangle$, coming from the $*$-product of $\frac{b_{0}}{L_{0}}\left|\phi_{0} * \phi_{0}\right\rangle$ with itself, in computing the solution to order $\widehat{\lambda}^{3}$. 
where

$$
\begin{aligned}
f_{3}(\omega, \hat{\lambda}) & =-\mathcal{N}^{-1} \widehat{\lambda}^{3}\left\langle\phi _ { 0 } \left|\left(\left|\phi_{0}\right\rangle * \frac{b_{0}}{L_{0}}\left|\phi_{0} * \phi_{0}\right\rangle+\frac{b_{0}}{L_{0}}\left|\phi_{0} * \phi_{0}\right\rangle *\left|\phi_{0}\right\rangle\right)\right.\right. \\
& =-2 \mathcal{N}^{-1} \widehat{\lambda}^{3}\left\langle\phi_{0} * \phi_{0}\left|\frac{b_{0}}{L_{0}}\right| \phi_{0} * \phi_{0}\right\rangle .
\end{aligned}
$$

This can be related to the four point amplitude $A^{(4)}$ in string field theory involving four external states $\left|\phi_{0}\right\rangle$. Taking into account the sum over $s, t$ and $u$ channel diagrams, and a factor of 2 coming from each vertex since the three point coupling in string field theory is accompanied by a factor of $1 / 3$ instead of $1 / 6$, we have'

$$
A^{(4)}(\omega)=12\left\langle\phi_{0} * \phi_{0}\left|\frac{b_{0}}{L_{0}}\right| \phi_{0} * \phi_{0}\right\rangle .
$$

Thus (3.24) can be written as

$$
f_{3}(\omega, \hat{\lambda})=-\frac{1}{6} \mathcal{N}^{-1} \widehat{\lambda}^{3} A^{(4)}(\omega) .
$$

$\omega_{3}$ is then the solution to the equation

$$
\left(\omega_{3}^{2}-m^{2}\right)=\frac{1}{6} \mathcal{N}^{-1} \widehat{\lambda}^{2} A^{(4)}\left(\omega_{3}\right) .
$$

To leading order we can set $\omega_{3}=m$ on the right hand side of (3.27) and get

$$
\omega_{3}=\sqrt{m^{2}+\frac{1}{6} \mathcal{N}^{-1} \widehat{\lambda}^{2} A^{(4)}(m)} .
$$

$\left|\Psi_{3}\right\rangle$ given in (3.23), evaluated at $\omega=\omega_{3}$, then gives the solution $\left|\Psi_{(3)}\right\rangle$ to order $\hat{\lambda}^{3}$.

This concludes our discussion on generating the time dependent solution of string field theory, describing the rolling of a tachyon away from its maximum. We shall end this section by mentioning three generalizations of this analysis:

- We have discussed the case where the tachyon begins rolling from an initial configuration where its time derivative vanishes and the field is displaced from its maximum. In this case the total energy of the system is less than that at the maximum. We could also consider the case where we begin with a configuration where the tachyon field is at its maximum and has a small velocity. The leading order solution in this case is proportional to $\sinh \left(\omega x^{0}\right)$ with $\omega=m$. As discussed in [3, 4, the full solution in this case can be found by making the replacement:

$$
x^{0} \rightarrow x^{0}+i \pi /(2 \omega), \quad \widehat{\lambda} \rightarrow-i \widehat{\lambda},
$$

in the solution we have obtained earlier.

\footnotetext{
${ }^{9}$ Throughout this paper $A^{(4)}$ will denote the amplitude in the euclidean string (field) theory.
} 
- The method discussed here can also be used to generate time dependent solutions describing oscillation of positive mass ${ }^{2}$ fields about the minimum of their potential. In this case there is no need for Wick rotation. For a field $\phi$ of mass $M$, we take the first order solution to be

$$
\widehat{\lambda} \cos \left(\omega X^{0}(0)\right) V_{\phi}(0) c_{1}|0\rangle
$$

where $V_{\phi}$ is the vertex operator for the zero momentum scalar. At leading order, $\omega=M$. We can now follow the iterative procedure described in this section to generate the solution to arbitrary power of $\hat{\lambda}$, and determine $\omega$ as a function of $\hat{\lambda}$ to that order. The $\hat{\lambda}$ dependence of $\omega$ will represent the anharmonicity of the oscillator due to the interaction terms in the string field theory action.

- One could consider generalizing the construction to the case where many tachyon fields roll simultaneously. In general, if we have $n$ scalar fields then for a two derivative action we have $2 n$ initial conditions. We shall now show that even in string field theory we can construct a $2 n$ parameter family of solutions describing the rolling of $n$ tachyons. To leading order the solution in the Wick rotated theory is:

$$
|\Psi\rangle=\sum_{i=1}^{n} \widehat{\lambda}^{(i)}\left|\phi_{0}^{(i)}\right\rangle,
$$

where

$$
\left|\phi_{0}^{(i)}\right\rangle=\cos \left(\omega^{(i)} X(0)+\varphi^{(i)}\right) V_{T}^{(i)}(0) c_{1}|0\rangle .
$$

$V_{T}^{(i)}$ is the vertex operator of the $i$ th tachyon with $\operatorname{mass}^{2}=-m_{(i)}^{2},\left\{\widehat{\lambda}^{(i)}\right\}$ and $\left\{\varphi^{(i)}\right\}$ are the $2 n$ parameters labelling the solution, and to leading order $\omega^{(i)}=m^{(i)}$. We shall assume that the $m^{(i)}$ 's are incomensurate. We now generate the higher order solutions by iterating (3.31) as in the case of a single tachyon field, keeping the coefficient of $\left|\phi_{0}^{(i)}\right\rangle$ held fixed at $\hat{\lambda}^{(i)}$ to all orders. The analog of $|\chi\rangle^{\prime}$ for a state $|\chi\rangle$ of ghost number 2 is defined as the projection of $|\chi\rangle$ in the subspace orthogonal to the one spanned by the states $\left|\phi_{0}^{(i)}\right\rangle$. Thus comparison of the coefficients of $c_{0}\left|\phi_{0}^{(i)}\right\rangle$ terms in the equation of motion determines $\omega^{(i)}$ as a function of the $\hat{\lambda}^{(j)}$ 's, whereas the comparison of the other terms in the equation of motion determine the component of $|\Psi\rangle$ in the subspace orthogonal to the one spanned by the $c_{0}\left|\phi_{0}^{(i)}\right\rangle$ 's. At the end of the computation we need to make the replacement $X \rightarrow-i X^{0}$, and $\varphi^{(i)} \rightarrow-i \theta^{(i)}$ where $\theta^{(i)}$ are taken to be real parameters.

For this procedure to work we need to ensure that the operator product of the operators $\cos \left(\omega^{(i)} X+\varphi^{(i)}\right) V_{T}^{(i)}$ in the matter sector does not generate another operator of dimension $=\mathcal{O}\left(\hat{\lambda}^{(i)}\right)$ outside the set $\left\{\cos \left(\omega^{(i)} X+\varphi^{(i)}\right) V_{T}^{(i)}\right\}$. The generic dangerous 
operators are $\sin \left(\omega^{(i)} X+\varphi^{(i)}\right) V_{T}^{(i)}$ and $\partial X$. The fact that $\sin \left(\omega^{(i)} X+\varphi^{(i)}\right) V_{T}^{(i)}$ is not generated can be seen as follows. If in a correlation function we have one insertion of $\sin \left(\omega^{(i)} X+\varphi^{(i)}\right)$ and multiple insertions of $\cos \left(\omega^{(j)} X+\varphi^{(j)}\right)$ for different $j$, then we can evaluate this by writing the sines and cosines as sum of exponentials. As long as the $\omega^{(j)}$ 's are incomensurate, to a correlator involving $\exp \left( \pm i\left(\omega^{(j)} X+\varphi^{(j)}\right)\right)$ will be non-zero only if the $\pm \omega^{(j)} X$ in the exponent cancel pairwise. As a result the accompanying $\pm \varphi^{(j)}$ also cancel pairwise. It is now easy to see that for each term in the correlator involving the $e^{i\left(\omega^{(i)} x+\varphi^{(i)}\right)}$ in $\sin \left(\omega^{(i)} x+\varphi^{(i)}\right)$, there is an equal and opposite contribution involving the $e^{-i\left(\omega^{(i)} x+\varphi^{(i)}\right)}$ term in $\sin \left(\omega^{(i)} x+\varphi^{(i)}\right)$, obtained by reversing the signs of the exponents of all the other terms in the correlator. $\square$ These two terms cancel. Thus such a correlator vanishes, implying in turn that the operator product of $\cos \left(\omega^{(j)} X+\varphi^{(j)}\right)$ does not contain a term proportional to $\sin \left(\omega^{(i)} X+\varphi^{(i)}\right)$.

To see the absence of $\partial X$ in the operator product of $\cos \left(\omega^{(j)} X+\varphi^{(j)}\right)$, we consider a correlation function with one insertion of $\partial X$ and multiple insertions of various factors of $\cos \left(\omega^{(j)} X+\varphi^{(j)}\right)$ on the boundary of the world-sheet. Since $\partial X$ can be thought of as the restriction of a bulk holomorphic current to the boundary, this contribution can be expressed as a sum of poles of $\partial X$ at the location of the various insertions of $\cos \left(\omega^{(j)} X+\varphi^{(j)}\right)$. The residue of the pole at the insertion of $\cos \left(\omega^{(k)} X+\varphi^{(k)}\right)$ is given by $\omega^{(k)} \sin \left(\omega^{(k)} X+\varphi^{(k)}\right)$. Thus

$$
\begin{aligned}
& \left\langle\partial X(z) \prod_{s=1}^{N} \cos \left(\omega^{\left(j_{s}\right)} X\left(t_{s}\right)+\varphi^{\left(j_{s}\right)}\right)\right\rangle \\
\propto & \sum_{r=1}^{N} \frac{\omega^{\left(j_{r}\right)}}{\left(z-t_{r}\right)}\left\langle\sin \left(\omega^{\left(j_{r}\right)} X\left(t_{r}\right)+\varphi^{\left(j_{r}\right)}\right) \prod_{s \neq r} \cos \left(\omega^{\left(j_{s}\right)} X\left(t_{s}\right)+\varphi^{\left(j_{s}\right)}\right)\right\rangle, \\
& 1 \leq j_{s} \leq n
\end{aligned}
$$

The result vanishes by previous argument. This, in turn, shows that the correlator of $\partial X$ with a set of $\cos \left(\omega^{(j)} X+\varphi^{(j)}\right)$ vanishes and hence we do not generate $\partial X$ in the operator product of $\cos \left(\omega^{(j)} X+\varphi^{(j)}\right)$.

Of course there may be other special dimension 1 operators which may be generated in the operator product of $\cos \left(\omega^{(j)} X+\varphi^{(j)}\right) V_{T}^{(j)}$. In that case the perturbative procedure outlined here for generating solutions of string field theory equations of motion breaks down.

\footnotetext{
${ }^{10}$ This restriction on $\omega^{(j)}$ means that the points in the parameter space where perturbation theory fails are dense in the full parameter space, but the situation here is no different from that in perturbation theory in ordinary Hamiltonian system.

${ }^{11}$ There are many ways to pair terms so that they cancel; here we only mention one of them.
} 


\section{Boundary Conformal Field Theory Description}

In this section we shall describe the BCFT associated with the solutions constructed in the previous section. Since in the euclidean theory the final solution is obtained by iterating an initial solution of the form

$$
\widehat{\lambda} \cos (\omega X(0)) V_{T}(0) c_{1}|0\rangle,
$$

we might expect that this corresponds to a BCFT that is obtained by perturbing the original BCFT by a boundary term

$$
\tilde{\lambda} \int d t \cos (\omega X(t)) V_{T}(t)
$$

where $\tilde{\lambda}=\hat{\lambda}+\mathcal{O}\left(\widehat{\lambda}^{2}\right)$ and $t$ is a parameter labelling the boundary of the world-sheet. In order for (4.2) to describe a BCFT the $\beta$-function of the theory must vanish. Since $\cos (\omega X) V_{T}$ has conformal weight $\left(\omega^{2}+h\right)=\left(\omega^{2}-m^{2}+1\right)$, the $\beta$ function for the coupling $\tilde{\lambda}$ has the form:

$$
\beta_{\tilde{\lambda}}=\left(\omega^{2}-m^{2}\right) \tilde{\lambda}+g(\omega, \tilde{\lambda}),
$$

where $g(\omega, \tilde{\lambda})$ represents higher order (in $\tilde{\lambda}$ ) contribution to the $\beta$-function. Thus the vanishing of the $\beta$ function requires: $\square^{2}$

$$
\left(\omega^{2}-m^{2}\right) \tilde{\lambda}+g(\omega, \tilde{\lambda})=0 .
$$

This is equivalent to the equation (3.12) in string field theory. Of course, in order to show that the perturbed theory is conformal we must ensure that the $\beta$-functions associated with all other operators also vanish. To this end we note that if $O$ denotes a boundary operator of dimension $h_{O}$, and if we add this operator with coefficient $\lambda_{O}$ in the perturbed theory, then the $\beta$-function of the coupling $\lambda_{O}$ is given by

$$
\beta_{O}=\left(h_{O}-1\right) \lambda_{O}+g_{O}\left(\tilde{\lambda}, \lambda_{O}, \ldots\right),
$$

where ... denote the coupling constants associated with the other operators added to the theory, and $g_{O}$ is the contribution to $\beta_{O}$ from higher order quantum corrections. Since $g_{O}$ is of order $\tilde{\lambda}^{2}$ or higher, as long as $\left(h_{O}-1\right)$ is of order 1 , we can make (4.5) vanish by choosing a $\lambda_{O}$ of order $\widetilde{\lambda}^{2}$. This procedure breaks down if $\left(h_{O}-1\right)$ is of order $\tilde{\lambda}$ or higher for some operator $O$ other than $\cos (\omega X) V_{T}$ (which has already been discussed in eq.(4.4)). Thus we require that the operator product of $\cos (\omega X) V_{T}$ with itself does not

\footnotetext{
${ }^{12}$ See [20] for a discussion on this. For application of the $\beta$-function equation to the study of tachyon condensation, see [22, 23].
} 
generate an operator of dimension $1+\mathcal{O}(\tilde{\lambda})$ other than $\cos (\omega X) V_{T}$. This is identical to the condition derived in the previous section for obtaining a solution of the string field theory equations of motion in a perturbation expansion in $\tilde{\lambda}$.

We shall now describe explicit computation of $\omega$ to order $\tilde{\lambda}^{2}$. For this we can set $\omega=m$ in the second term in (4.4). This gives

$$
\omega^{2}=m^{2}-\tilde{\lambda}^{-1} g(m, \tilde{\lambda}) .
$$

Thus we need to compute $g(m, \tilde{\lambda})$. For this computation we can pretend that the $X$ coordinate is compactified with period $2 \pi / m$. In that case $\cos (m X) V_{T}$, being a dimension 1 operator, represents a massless scalar field $\varphi$ in this auxiliary theory. Let $\mathcal{V}(\varphi)$ denote the tree level effective potential for this scalar field obtained after integrating out all the other fields. (For $\omega \neq m$, there is also a 'mass term' $\frac{1}{2}\left(\omega^{2}-m^{2}\right) \varphi^{2}$ coming from the dimension of the operator $\cos (\omega X) V_{T}$.) Then by the usual correspondence between the equations of motion of string theory and the condition of conformal invariance, we can compute the $\beta$-function $g(m, \tilde{\lambda})$ as

$$
g(m, \tilde{\lambda})=\mathcal{V}^{\prime}(\tilde{\lambda})
$$

for suitably normalized $\mathcal{V}$. In order to compute $\mathcal{V}(\varphi)$ we simply compute the tree level amplitude involving the massless scalars $\varphi$ in this auxiliary string theory. First of all it is clear that there is no $\varphi^{3}$ term in $\mathcal{V}(\varphi)$, since the three point amplitude of the scalar field described by the vertex operator $\cos (m X) V_{T}$ vanishes due to $X$-momentum conservation. Thus the leading contribution to $\mathcal{V}(\varphi)$ comes at the quartic order. The coefficient of this term can be identified as $-\left(\mathcal{N}^{-1} / 4\right.$ !) times the on-shell four point amplitude $A^{(4)}$ of the auxiliary scalar field at zero momentum:

$$
\mathcal{V}(\varphi)=-\left(\mathcal{N}^{-1} / 4 !\right) A^{(4)} \varphi^{4}+\mathcal{O}\left(\varphi^{6}\right)
$$

where $\mathcal{N}$ is the BPZ norm of the state $\cos (m X(0)) V_{T}(0)|0\rangle$ in the matter sector. ${ }^{\mathrm{T}} \mathrm{T}$ Thus we get, from (4.7),

$$
g(m, \widetilde{\lambda})=-\left(\mathcal{N}^{-1} / 6\right) A^{(4)} \widetilde{\lambda}^{3}+\mathcal{O}\left(\widetilde{\lambda}^{5}\right)
$$

and hence from (4.6)

$$
\omega^{2}=m^{2}+\frac{\mathcal{N}^{-1}}{6} A^{(4)} \tilde{\lambda}^{2}+\mathcal{O}\left(\widetilde{\lambda}^{4}\right) .
$$

This of course agrees with eq.(3.28) derived from string field theory.

\footnotetext{
${ }^{13}$ The simplest way to keep track of the normalization factor $\mathcal{N}$ is to first do the computation by assuming $\cos (m X) V_{T}$ to be a normalized operator, and then account for the normalization by replacing $A^{(4)}$ by $\mathcal{N}^{-2} A^{(4)}$ and $\widetilde{\lambda}$ by $\mathcal{N}^{1 / 2} \widetilde{\lambda}$ in $(4.10)$.
} 
At this stage we should mention one subtle point. Interpreting the perturbed theory (4.2) as a deformed BCFT in the sense of renormalization group flow makes sense strictly if the operator $\cos (\omega X) V_{T}$ is a relevant perturbation, 1.e. if $\omega^{2}<m^{2}$. From (4.4), (4.7) we see that this requires $g(\omega, \tilde{\lambda})$, or equivalently $\mathcal{V}(\varphi)$, to be positive near $\varphi=0$. In the analysis of string field theory in section (3) we did not encounter such a constraint. If $\mathcal{V}$ turns out to be negative, we can still find a solution in string field theory by choosing $\omega^{2}-m^{2}$ to be positive. This will correspond to a net potential for the auxiliary scalar field $\varphi$ which has a minimum at $\varphi=0$ and a maximum at a nearby point, and the non-trivial solution of (4.4) will correspond to the maximum of the potential. Thus even for negative $\mathcal{V}$, we should expect a family of BCFT labelled by $\tilde{\lambda}$, although members of this family are not obtained as deformation of the original BCFT by a relevant perturbation. Rather, the original BCFT is obtained as a relevant deformation of each member of this family.

When $\omega^{2}<m^{2}$ so that the perturbing operator is relevant, it is more natural to treat $\omega$ rather than $\lambda$ as the independent parameter. We simply deform the theory by the operator $\cos (\omega X) V_{T}$, and consider the boundary CFT to which the theory flows in the infrared. This will give a family of boundary CFT's labeled by $\omega$. Inverse Wick rotation of these boundary CFT's then give us the family of time dependent solutions corresponding to different initial conditions.

As special examples of the general class of perturbed BCFT's discussed above, we can consider the case where $g(m, \tilde{\lambda})$ vanishes for arbitrary $\tilde{\lambda}$, and as a result (4.4) is satisfied for $\omega=m$ for all $\tilde{\lambda}$. In this case the operator $\cos (m X) V_{T}$ is an exactly marginal operator.

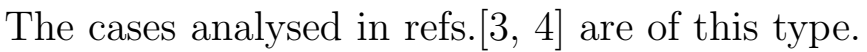

Given a BCFT obtained by perturbing the euclidean theory by the operator (4.2), we can construct a BCFT by the inverse Wick rotation of this perturbed theory. This will correspond to adding a perturbation

$$
\tilde{\lambda} \cosh \left(\omega X^{0}(0)\right) V_{T}(0) c_{1}|0\rangle,
$$

to the world-sheet theory with Minkowski signature space-time. The boundary state 24 associated with this BCFT, containing information about the time evolution of the energymomentum tensor 25, 4, can be obtained from Wick rotation of the boundary state associated with the euclidean theory. Time evolution of the sources of other massless closed string fields, e.g. the dilaton, the anti-symmetric tensor field $B_{\mu \nu}$, and various Ramond-Ramond fields, can also be extracted from the boundary state.

To see more explicitly how we can extract the energy-momentum tensor from the boundary state, we recall that [4] if part of the boundary state involving oscillators of level $(1,1)$ acting on the ghost number 3 vacuum has the form

$$
\int d^{26} k\left[\widetilde{A}_{\mu \nu}(k) \alpha_{-1}^{\mu} \bar{\alpha}_{-1}^{\nu}+\widetilde{B}(k)\left(\bar{b}_{-1} c_{-1}+b_{-1} \bar{c}_{-1}\right)\right]\left(c_{0}+\bar{c}_{0}\right) c_{1} \bar{c}_{1}|k\rangle,
$$


then the energy momentum tensor $T_{\mu \nu}$ is given by:

$$
T_{\mu \nu}(x)=K\left(A_{\mu \nu}(x)+\eta_{\mu \nu} B(x)\right),
$$

where $A_{\mu \nu}(x)$ and $B(x)$ are Fourier transforms of $\widetilde{A}_{\mu \nu}(k)$ and $\widetilde{B}(k)$ respectively, and $K$ is a $\tilde{\lambda}$-independent constant. In the Wick rotated theory the energy momentum tensor computed using this procedure will have the form:

$$
T_{\mu \nu}(x, \vec{x})=\sum_{n=0}^{\infty} T_{\mu \nu}^{(n)}(\vec{x}) \cos (n \omega x)
$$

where $\vec{x}$ denotes the spatial coordinates of the original theory. Then after Wick rotation we have:

$$
\begin{aligned}
T_{00}\left(x^{0}, \vec{x}\right) & =-\sum_{n=0}^{\infty} T_{x x}^{(n)}(\vec{x}) \cosh \left(n \omega x^{0}\right), \\
T_{0 i}\left(x^{0}, \vec{x}\right) & =i \sum_{n=0}^{\infty} T_{x i}^{(n)}(\vec{x}) \cosh \left(n \omega x^{0}\right), \\
T_{i j} & =\sum_{n=0}^{\infty} T_{i j}^{(n)}(\vec{x}) \cosh \left(n \omega x^{0}\right) .
\end{aligned}
$$

Since in the Wick rotated theory the sum over $n$ in (4.14) is expected to converge and generate a finite energy-momentum tensor, we expect that after Wick rotation the sum

over $n$ in (4.15) will converge for sufficiently small $x^{0}$ and $\tilde{\lambda}$. We do expect the sum to diverge for large $x^{0}$, and checking whether the results for small $x^{0}$ can be analytically continued beyond the range of convergence or not, we shall have to determine whether during the time evolution the energy momentum tensor hits a real singularity at a finite value of $x^{0}$.

In principle, we should also be able to extract the energy-momentum tensor from the solution of the string field theory equations of motion constructed in the previous section, since this solution is expected to have all the information about the deformed boundary CFT. At present however it is not known how to do this.

\section{Explicit Example}

In this section we shall discuss an explicit example of the construction described in the previous section. The system that we shall consider is a D-p-brane of bosonic string theory, with one direction wrapped on a circle of radius $R>1$. This has the usual tachyonic mode of $\operatorname{mass}^{2}=-1$, whose time evolution was discussed in [3]. But this also has another tachyonic mode of mass ${ }^{2}$

$$
R^{-2}-1 \equiv-m^{2}
$$


coming from the first momentum mode of the standard tachyon along the circle. If we denote by $y$ the coordinate along the circle, then the matter sector vertex operator associated with this tachyonic mode (with zero momentum along the non-compact directions) can be taken to be:

$$
V_{T}=\cos (Y / R) .
$$

We shall discuss the rolling of this tachyon when it is displaced from its maximum. As usual we denote by $x$ the Wick rotated time coordinate. The conformal field theory associated with the (Wick rotated) rolling tachyon solution is obtained by perturbing the original free conformal field theory by the boundary operator

$$
\tilde{\lambda} \int d t \cos (\omega X(t)) \cos (Y(t) / R) \text {. }
$$

Eq.(4.4) now takes the form:

$$
\left(\omega^{2}+R^{-2}-1\right) \tilde{\lambda}+g(\omega, \tilde{\lambda})=0 .
$$

To leading order the solution to this equation is $\omega=m=\sqrt{1-R^{-2}}$. From eq.(4.10) we see that the computation of $\mathcal{O}\left(\widetilde{\lambda}^{2}\right)$ correction to $\omega$ requires us to compute $A^{(4)}$ involving four external states associated with the matter sector vertex operator $\cos (m X) \cos (Y / R)$. This is the task to which we shall now turn.

Expressing the scalar field vertex operator as

$$
\cos (m X) \cos (Y / R)=\frac{1}{4}\left(e^{i m X}+e^{-i m X}\right)\left(e^{i Y / R}+e^{-i Y / R}\right),
$$

we can relate the four point amplitude $A^{(4)}$ of the scalar field to the Veneziano amplitude involving external states associated with matter sector vertex operators $e^{ \pm i m X} e^{ \pm i Y / R}$. Since the right hand side of eq.(5.5) has four terms, there are altogether $4^{4}=256$ terms involved in the computation of the four point amplitude $A^{(4)}$. However all but 36 of them vanish by $X$ - or $Y$-momentum conservation. 12 of the non-vanishing terms are each given by $\frac{1}{4^{4}} V(s=-4, t=0)$, and each of the rest 24 terms is given by $\frac{1}{4^{4}} V\left(s=-4 R^{-2}, t=\right.$ $\left.-4\left(1-R^{-2}\right)\right)$, where $V(s, t)$ is the Veneziano amplitude

$$
\begin{gathered}
V(s, t)=2\left(\frac{\Gamma(-1-s) \Gamma(-1-t)}{\Gamma(-2-s-t)}+\frac{\Gamma(-1-s) \Gamma(-1-u)}{\Gamma(-2-s-u)}+\frac{\Gamma(-1-u) \Gamma(-1-t)}{\Gamma(-2-u-t)}\right) \\
u=-4-s-t .
\end{gathered}
$$

Thus we get

$$
\begin{aligned}
A^{(4)} & =\frac{1}{4^{4}}\left(12 V(s=-4, t=0)+24 V\left(s=-4 R^{-2}, t=-4\left(1-R^{-2}\right)\right)\right. \\
& =-\frac{3}{32}\left(R^{2}-2\right)\left[4 R^{-2} F^{\prime}\left(4 R^{-2}\right) / F\left(4 R^{-2}\right)+F\left(4 R^{-2}\right)-1\right],
\end{aligned}
$$


where

$$
F(x)=\Gamma(1-x) \Gamma(1+x)=\frac{\pi x}{\sin (\pi x)} .
$$

Using eqs.(4.10), (5.7), and the fact that $\mathcal{N}=1 / 4$ for the operator (5.5), we now get

$$
\omega^{2}=1-R^{-2}-\frac{1}{16}\left(R^{2}-2\right)\left[4 R^{-2} F^{\prime}\left(4 R^{-2}\right) / F\left(4 R^{-2}\right)+F\left(4 R^{-2}\right)-1\right] \widetilde{\lambda}^{2}+\mathcal{O}\left(\widetilde{\lambda}^{4}\right)
$$

This determines the value of $\omega$ to order $\widetilde{\lambda}^{2}$ for which perturbation by the vertex operator $\cos (\omega X) \cos (Y / R)$ generates a boundary CFT. The time dependent solution describing the rolling tachyon is then determined by the inverse Wick rotation $X=-i X^{0}$ of this solution. In particular by inverse Wick rotating the boundary state associated with the perturbed euclidean BCFT, we can determine the energy-momentum tensor associated with the time dependent solution.

Unfortunately for generic $R$ the perturbed BCFT is not solvable, and hence we cannot explicitly compute the boundary state associated with this BCFT. For $R \rightarrow \infty$ the amplitude given in eq. (5.7) vanishes. This is consistent with the fact that in this limit the perturbing operator $\cos (X)$ is exactly marginal and hence the corresponding $\beta$-function must vanish. The associated conformal field theory has been discussed in detail in [26]

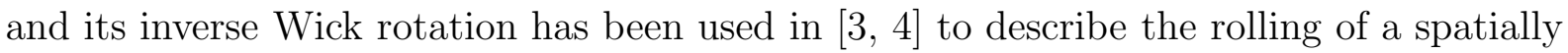
homogeneous tachyon field on the D-brane. $A^{(4)}$ also vanishes at $R=1$ where the perturbing operator is $\cos (Y)$. There is another value of $R$ where $A^{(4)}$ vanishes, namely at $R=\sqrt{2}$. Thus one might expect that the corresponding perturbation becomes exactly marginal at this point. To see that this is indeed the case, let us note that at $\omega=1 / \sqrt{2}$, the perturbing operator is given by:

$\tilde{\lambda} \int d t \cos (X(t) / \sqrt{2}) \cos (Y(t) / \sqrt{2})=\frac{\tilde{\lambda}}{2}[\cos ((X(t)+Y(t)) / \sqrt{2})+\cos ((X(t)-Y(t)) / \sqrt{2})]$.

This corresponds to perturbing the theory by a sum of two commuting exactly marginal deformations, and hence represents a marginal deformation. The associated BCFT is exactly solvable. In fact, defining

$$
Z^{1}=(X+Y) / \sqrt{2}, \quad Z^{2}=(X-Y) / \sqrt{2},
$$

we see that the net boundary state is given by the product

$$
|\mathcal{B}\rangle_{Z^{1}} \otimes|\mathcal{B}\rangle_{Z^{2}} \otimes|\mathcal{B}\rangle_{c=24} \otimes|\mathcal{B}\rangle_{\text {ghost }}
$$

\footnotetext{
${ }^{14}$ Note that $F\left(4 R^{-2}\right)$ diverges at $R=\sqrt{2}$ and so in evaluating (5.9) for $R=\sqrt{2}$ we need to carefully take the limit. Nevertheless the right hand side of (5.9) can be shown to vanish at $R=\sqrt{2}$.
} 
$|\mathcal{B}\rangle_{c=24}$ denotes the boundary state associated with the 24 free scalar fields, and $|\mathcal{B}\rangle_{\text {ghost }}$ denotes the boundary state associated with the ghost fields. These are given by their free field expressions. On the other hand the relevant part of the boundary states $|\mathcal{B}\rangle_{Z^{1}}$ and $|\mathcal{B}\rangle_{Z^{2}}$, associated with the perturbed BCFT associated with the scalar fields $Z^{1}$ and $Z^{2}$, can be read out from the results of [26, 27, 28, 价. If we consider for simplicity the case of the D-25-brane, the part of the boundary state, relevant for the computation of the energy-momentum tensor, is proportional to:

$$
\begin{aligned}
& {\left[f((i X(0)+i Y(0)) / \sqrt{2})-\frac{1}{2}\left(\alpha_{-1}^{X}+\alpha_{-1}^{Y}\right)\left(\bar{\alpha}_{-1}^{X}+\bar{\alpha}_{-1}^{Y}\right) g((i X(0)+i Y(0)) / \sqrt{2})\right]} \\
& {\left[f((i X(0)-i Y(0)) / \sqrt{2})-\frac{1}{2}\left(\alpha_{-1}^{X}-\alpha_{-1}^{Y}\right)\left(\bar{\alpha}_{-1}^{X}-\bar{\alpha}_{-1}^{Y}\right) g((i X(0)-i Y(0)) / \sqrt{2})\right]} \\
& {\left[1-\alpha_{-1}^{i} \bar{\alpha}_{-1}^{i}\right]\left[1-\bar{b}_{-1} c_{-1}-b_{-1} \bar{c}_{-1}\right]\left(c_{0}+\bar{c}_{0}\right) c_{1} \bar{c}_{1}|0\rangle .}
\end{aligned}
$$

Here $\alpha_{-1}^{i}, \bar{\alpha}_{-1}^{i}$ for $2 \leq i \leq 25$ are the oscillators associated with the spectator bosons $X^{i}$, $\alpha_{-1}^{X}, \bar{\alpha}_{-1}^{X}, \alpha_{-1}^{Y}, \bar{\alpha}_{-1}^{Y}$ are the oscillators associated with the scalars $X$ and $Y$, and $b_{n}, c_{n}$, $\bar{b}_{n}, \bar{c}_{n}$ are the oscillators associated with the ghost fields $b, c, \bar{b}, \bar{c}$. The functions $g(x)$ and $f(x)$ have been given in [3, 团:

$$
f\left(x^{0}\right)=\frac{1}{1+e^{x^{0}} \sin (\widetilde{\lambda} \pi / 2)}+\frac{1}{1+e^{-x^{0}} \sin (\widetilde{\lambda} \pi / 2)}-1, \quad g\left(x^{0}\right)=\cos (\widetilde{\lambda} \pi)+1-f\left(x^{0}\right) .
$$

Note that these formulæ differ from that of [3, [4 by a replacement $\tilde{\lambda} \rightarrow \tilde{\lambda} / 2$, whose origin can be traced to the explicit factor of $1 / 2$ multiplying $\tilde{\lambda}$ on the right hand side of (5.10).

We can now make an inverse Wick rotation $X=-i X^{0}$ to get the boundary state in the Minkowski signature theory:

$$
\begin{aligned}
& {\left[f\left(\left(X^{0}(0)+i Y(0)\right) / \sqrt{2}\right)-\frac{1}{2}\left(-i \alpha_{-1}^{0}+\alpha_{-1}^{Y}\right)\left(-i \bar{\alpha}_{-1}^{0}+\bar{\alpha}_{-1}^{Y}\right) g\left(\left(X^{0}(0)+i Y(0)\right) / \sqrt{2}\right)\right]} \\
& {\left[f\left(\left(X^{0}(0)-i Y(0)\right) / \sqrt{2}\right)-\frac{1}{2}\left(-i \alpha_{-1}^{0}-\alpha_{-1}^{Y}\right)\left(-i \bar{\alpha}_{-1}^{0}-\bar{\alpha}_{-1}^{Y}\right) g\left(\left(X^{0}(0)-i Y(0)\right) / \sqrt{2}\right)\right]} \\
& {\left[1-\alpha_{-1}^{i} \bar{\alpha}_{-1}^{i}\right]\left[1-\bar{b}_{-1} c_{-1}-b_{-1} \bar{c}_{-1}\right]\left(c_{0}+\bar{c}_{0}\right) c_{1} \bar{c}_{1}|0\rangle .}
\end{aligned}
$$

Comparing (5.15) with (4.12) we get,

$$
\begin{aligned}
& A_{00}=\frac{1}{2}\left[g\left(\left(x^{0}+i y\right) / \sqrt{2}\right) f\left(\left(x^{0}-i y\right) / \sqrt{2}\right)+g\left(\left(x^{0}-i y\right) / \sqrt{2}\right) f\left(\left(x^{0}+i y\right) / \sqrt{2}\right)\right], \\
& A_{0 y}=A_{y 0}=\frac{i}{2}\left[g\left(\left(x^{0}+i y\right) / \sqrt{2}\right) f\left(\left(x^{0}-i y\right) / \sqrt{2}\right)-g\left(\left(x^{0}-i y\right) / \sqrt{2}\right) f\left(\left(x^{0}+i y\right) / \sqrt{2}\right)\right], \\
& A_{y y}=-\frac{1}{2}\left[g\left(\left(x^{0}+i y\right) / \sqrt{2}\right) f\left(\left(x^{0}-i y\right) / \sqrt{2}\right)+g\left(\left(x^{0}-i y\right) / \sqrt{2}\right) f\left(\left(x^{0}+i y\right) / \sqrt{2}\right)\right],
\end{aligned}
$$




$$
\begin{aligned}
A_{i j} & =-\delta_{i j} f\left(\left(x^{0}+i y\right) / \sqrt{2}\right) f\left(\left(x^{0}-i y\right) / \sqrt{2}\right) \\
B & =-f\left(\left(x^{0}+i y\right) / \sqrt{2}\right) f\left(\left(x^{0}-i y\right) / \sqrt{2}\right) .
\end{aligned}
$$

Using (4.13), (5.14) and (5.16) we get

$$
\begin{aligned}
T_{00} & =K\left(A_{00}-B\right)=\frac{K}{2}(\cos (\widetilde{\lambda} \pi)+1)\left[f\left(\left(x^{0}+i y\right) / \sqrt{2}\right)+f\left(\left(x^{0}-i y\right) / \sqrt{2}\right)\right] \\
T_{0 y} & =K A_{0 y}=\frac{i K}{2}(\cos (\widetilde{\lambda} \pi)+1)\left[f\left(\left(x^{0}-i y\right) / \sqrt{2}\right)-f\left(\left(x^{0}+i y\right) / \sqrt{2}\right)\right] \\
T_{y y} & =K\left(A_{y y}+B\right)=-\frac{K}{2}(\cos (\widetilde{\lambda} \pi)+1)\left[f\left(\left(x^{0}+i y\right) / \sqrt{2}\right)+f\left(\left(x^{0}-i y\right) / \sqrt{2}\right)\right], \\
T_{i j} & =K\left(A_{i j}+B \delta_{i j}\right)=-2 K \delta_{i j} f\left(\left(x^{0}+i y\right) / \sqrt{2}\right) f\left(\left(x^{0}-i y\right) / \sqrt{2}\right)
\end{aligned}
$$

All other components of $T_{\mu \nu}$ vanish. From these expressions one can easily verify the conservation laws:

$$
\partial_{0} T_{00}-\partial_{y} T_{0 y}=0, \quad \partial_{0} T_{0 y}-\partial_{y} T_{y y}=0 .
$$

If we consider a general D-p-brane with $p<25$, then $T_{i j}$ associated with the $(25-p)$ transverse directions vanish. Furthermore the non-vanishing components of $T_{\mu \nu}$ are each multiplied by a delta function involving the transverse coordinates. The $\tilde{\lambda}$ independent constant $K$ is computed by requiring that for $\widetilde{\lambda}=0, T_{00}$ should reproduce the tension $\mathcal{T}_{p}$ of a D-p-brane. This gives

$$
K=\frac{\mathcal{T}_{p}}{2}
$$

From (5.14) we get

$$
f\left(\left(x^{0} \pm i y\right) / \sqrt{2}\right)=\frac{1}{1+e^{x^{0} / \sqrt{2}} e^{ \pm i y / \sqrt{2}} \sin (\widetilde{\lambda} \pi / 2)}+\frac{1}{1+e^{-x^{0} / \sqrt{2}} e^{\mp i y / \sqrt{2}} \sin (\widetilde{\lambda} \pi / 2)}-1
$$

Thus $f\left(\left(x^{0} \pm i y\right) / \sqrt{2}\right)$ and hence the energy momentum tensor hits a singularity at

$$
x^{0}=\sqrt{2} \ln \left|\frac{1}{\sin (\widetilde{\lambda} \pi / 2)}\right| \equiv x_{c}^{0}, \quad y= \begin{cases}\sqrt{2} \pi, & \text { for } \quad \tilde{\lambda}>0 \\ 0, & \text { for } \quad \tilde{\lambda}<0 .\end{cases}
$$

In particular the energy-density at the singular point approaches $\infty$. Thus we cannot naively evolve the system beyond this time using the classical open string field equations.

It is instructive to examine the nature of the singularity in a little more detail. Using eqs. (5.17), (5.20) we get the total energy of the system to be:

$$
\mathcal{E}=\int_{0}^{2 \pi \sqrt{2}} d y T_{00}=2 \pi \sqrt{2} K(1+\cos (\widetilde{\lambda} \pi))
$$




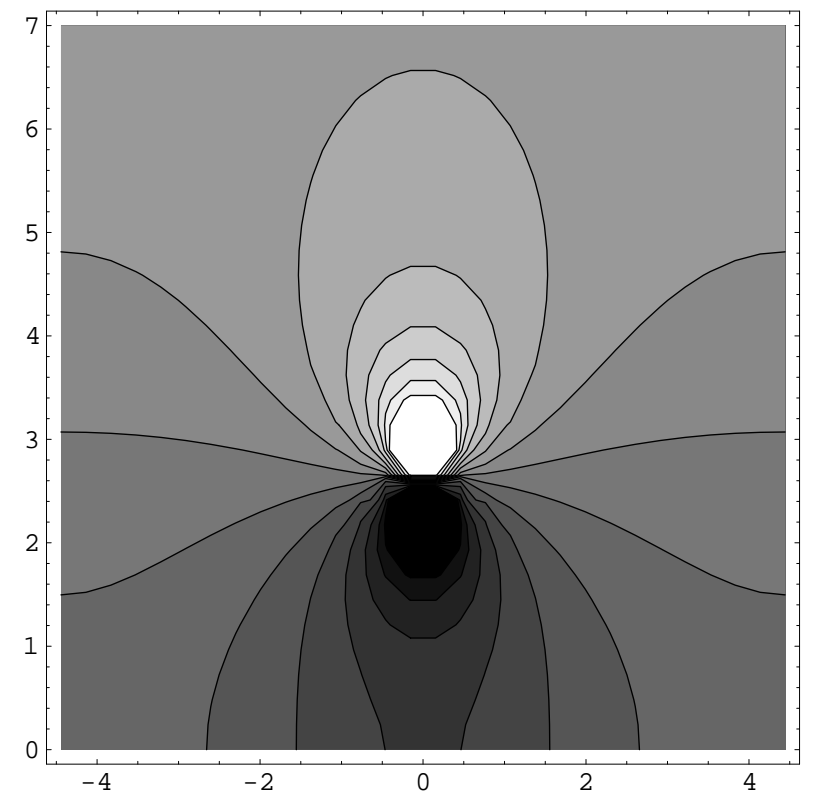

Figure 2: The contour plot of the time evolution of the energy density $T_{00}$ computed from eq. (5.17), (5.20) for $\tilde{\lambda}=-0.1$. The horizontal axis is the $y$ axis, the vertical axis is the time $\left(x^{0}\right)$ axis, and the shading at a given point $(y, t)$ shows the value of $T_{00}$, with darker shade representing higher energy density. We see from this that the $T_{00}$ hits a singularity at $x^{0} \simeq 2.62, y=0$. At this space-time point, the system loses a net amount of energy equal to the total initial energy of the system. Immediately after this singularity the energy density becomes negative around $y=0$, as shown by the lighter regions, and eventually the energy density approaches zero everywhere.

for $x^{0}<x_{c}^{0}$. If we naively continue the expression for $T_{00}$ to $x^{0}>x_{c}^{0}$, we find that the total energy vanishes. In order to see the origin of this 'violation' of energy conservation, we can examine the form of the singularity at $x^{0}=x_{c}^{0}, y=0$ (concentrating for definiteness on the $\tilde{\lambda}<0$ case.) Defining

$$
\Theta \equiv e^{\left(x^{0}-x_{c}^{0}\right) / \sqrt{2}}=-e^{x^{0} / \sqrt{2}} \sin (\widetilde{\lambda} \pi) \simeq 1
$$

near the singularity, the singular part of $T_{00}$ near $(1-\Theta) \sim y \simeq 0$ is given by:

$$
\left(T_{00}\right)_{\text {singular }}=K(1+\cos (\widetilde{\lambda} \pi)) \frac{(1-\Theta)}{(1-\Theta)^{2}+\frac{1}{2} y^{2}} \simeq \operatorname{sgn}(1-\Theta) \pi \sqrt{2} K(1+\cos (\widetilde{\lambda} \pi)) \delta(y) .
$$

Since $(1-\Theta)$ changes sign as $x^{0}$ passes from below to above $x_{c}^{0}$, there is a net loss of energy at $y=0$ at $x^{0}=x_{c}^{0}$, The amount of energy lost at $y=0$ as $x^{0}$ passes from below 
$x_{c}^{0}$ to above $x_{c}^{0}$ is $2 \pi \sqrt{2} K(1+\cos (\tilde{\lambda} \pi))$, - same as the total energy of the system. As a result the average of the energy density in the rest of the system must vanish after the critical time $x_{c}^{0}$, as has already been seen in explicit calculation. If we continue the formula for $T_{00}$ beyond this critical time, the system eventually evolves to a configuration with zero energy density everywherem There is an apparent violation of energy conservation at $x^{0}=x_{c}^{0}$, but the most conservative interpretation of this is simply that we create a codimension 1 D-brane at $y=0$, with a delta function source of energy that is not visible in the formula (5.17). The total energy stored at $y=0$ does not agree with the tension of a codimension 1 D-brane; in particular the ratio of the total energy of the system to that of a codimension 1 D-brane is given by:

$$
\frac{1}{\sqrt{2}}(1+\cos (\pi \tilde{\lambda}))
$$

However the final state could represent a state of the codimension 1 D-brane with nontrivial time dependent tachyon configuration, with the excess (deficit) energy stored in the tachyon field. Fig. 2 shows the time evolution of $T_{00}$ given in eqs.(5.17), (5.20) for a specific value of $\tilde{\lambda}$.

\section{Time Dependent Solutions on D-branes in Super- string Theories}

The analysis of the previous sections can be easily generalized to the case of superstring theories. Let us consider a generic D-brane system with a tachyonic mode, and let the zero momentum tachyon vertex operator have the form $e^{-\phi} V_{T}^{(-1)}$ in the -1 picture 29], where $\phi$ denotes the bosonized ghost field and $V_{T}^{(-1)}$ denotes the matter part of the vertex operator. If $V_{T}^{(-1)}$ has dimension $h<1 / 2$, then the tachyon has mass ${ }^{2}=\left(h-\frac{1}{2}\right) \equiv-m^{2}$ and at the linearized level the time dependent tachyon solution takes the form $\tilde{\lambda} \cosh \left(m x^{0}\right)$. In the Wick rotated theory this corresponds to $\tilde{\lambda} \cos (m x)$, and amounts to perturbing the BCFT by a term

$$
\tilde{\lambda} \int d t d \theta \cos (m X(t, \theta)) V_{T}^{(-1)}(t, \theta),
$$

where $t$ denotes the coordinate labelling the world-sheet boundary, $\theta$ is the fermionic coordinate on the superspace, and we consider $X$ and $V_{T}^{(-1)}$ as superfields. Although to lowest order in $\tilde{\lambda}$ this represents a marginal deformation, in general once higher order corrections are taken into account this no longer represents a marginal deformation. We

\footnotetext{
${ }^{15}$ Of course, near the singularity $x^{0}=x_{c}^{0}, y=0$, the back reaction of closed string fields will be important, and a realistic analysis of the dynamics must include this effect.
} 
remedy this by modifying (6.1) to

$$
\tilde{\lambda} \int d t d \theta \cos (\omega X(t, \theta)) V_{T}^{(-1)}(t, \theta)
$$

where $\omega$ is a constant. The full $\beta$-function associated with the coupling $\tilde{\lambda}$ now takes the form:

$$
\omega_{\tilde{\lambda}}=\left(\omega^{2}-m^{2}\right) \widetilde{\lambda}+g(\omega, \widetilde{\lambda}),
$$

as in (4.3). We determine $\omega$ for a given $\tilde{\lambda}$ by demanding that $\beta_{\widetilde{\lambda}}$ vanishes. This gives:

$$
\left(\omega^{2}-m^{2}\right) \tilde{\lambda}+g(\omega, \tilde{\lambda})=0
$$

This determines $\omega$ as a function of $\tilde{\lambda}$. Thus for example the leading correction to $\omega$ is given by

$$
\omega^{2} \simeq m^{2}-g(m, \tilde{\lambda}) / \widetilde{\lambda}
$$

The $\beta$-functions associated with the other operators can be made to vanish as in section 4 provided the product of $(6.2)$ with itself does not generate integral of any superfield of dimension $1 / 2+\mathcal{O}(\tilde{\lambda})$ other than the superfield $\cos (\omega X(t, \theta)) V_{T}^{(-1)}(t, \theta)$ itself.

For an explicit example we can consider the case analogous to the one discussed in section 5 for open bosonic string theory. We take a non-BPS D-p-brane with one direction compactified on a circle of radius $>\sqrt{2}$. Let $y$ denote this compact direction. In this case the first momentum mode of the tachyon along the circle, described by the wave-function $\cos (y / R)$, can be thought of as a scalar field living in $((p-1)+1)$ dimensions with mass ${ }^{2}$ :

$$
\frac{1}{R^{2}}-\frac{1}{2} \equiv-m^{2}
$$

Displacing this tachyonic mode from its maximum and letting it roll amounts to displacing the full $(p+1)$ dimensional tachyon from its maximum by an amount proportional to $\cos (y / R)$ and let it roll. Since the sign of the displacement is different for different values of $y$, we expect that the time evolution of the tachyon will produce a kink-antikink pair on the D-p-brane world volume, centered around $y= \pm \pi R / 2$.

We shall study the time evolution by viewing this particular mode of the tachyon as a scalar field in $((p-1)+1)$ dimensions. The vertex operator of this zero momentum tachyon in the -1 picture is $e^{-\phi} \cos (Y / R) \otimes \sigma_{1}$ where $\sigma_{1}$ is the Chan-Paton factor 30, 12. Thus in order to construct the Wick rotated version of the rolling tachyon solution, we need to perturb the BCFT by the operator

$$
\tilde{\lambda} \int d t d \theta \cos (\omega X(t, \theta)) \cos (Y(t, \theta) / R) \otimes \sigma_{1}
$$


If we expand the superfields $X(t, \theta)$ and $Y(t, \theta)$ as

$$
X(t, \theta)=X(t)+\theta \psi_{x}(t), \quad Y(t, \theta)=Y(t)+\theta \psi_{y}(t)
$$

where $\psi_{x}$ and $\psi_{y}$ are the world-sheet fermionic partners of the $X$ and the $Y$ fields respectively, then the perturbation can be expressed as

$$
-\tilde{\lambda} \int d t\left[\omega \sin (\omega X(t)) \cos (Y(t) / R)+R^{-1} \cos (\omega X(t)) \sin (Y(t) / R)\right] \otimes \sigma_{1} .
$$

As in section 5 we can relate the computation of $g(m, \widetilde{\lambda})$ appearing in eq. (6.5) to leading order in $\tilde{\lambda}$ to the computation of the four point amplitude of four on-shell external states, each described the the vertex operator $e^{-\phi} \cos (m X) \cos (Y / R) \otimes \sigma_{1}$. This in turn will determine the leading correction to $\omega$ via eq.6.5). We shall not carry out the detailed computation here.

We can consider the special value of the radius $R=2$. In this case $m=1 / 2$, and to leading order when we set $\omega=m$ the perturbation (6.7) takes the form:

$$
\begin{aligned}
& \tilde{\lambda} \int d t d \theta \cos (X(t, \theta) / 2) \cos (Y(t, \theta) / 2) \otimes \sigma_{1} \\
= & \frac{\tilde{\lambda}}{2} \int d t d \theta\left[\cos \left(Z_{1}(t, \theta) / \sqrt{2}\right)+\cos \left(Z_{2}(t, \theta) / \sqrt{2}\right)\right] \otimes \sigma_{1},
\end{aligned}
$$

where

$$
Z_{1}=(X+Y) / \sqrt{2}, \quad Z_{2}=(X-Y) / \sqrt{2}
$$

Writing

$$
Z_{i}(t, \theta)=Z_{i}(t)+\theta \psi_{i}(t)
$$

we can rewrite the perturbation as

$$
-\frac{\tilde{\lambda}}{2 \sqrt{2}} \int d t\left[\psi_{1}(t) \sin \left(Z_{1}(t) / \sqrt{2}\right)+\psi_{2}(t) \sin \left(Z_{2}(t) / \sqrt{2}\right)\right] \otimes \sigma_{1}
$$

Following the results of [30, 31] one can show that each of the two terms in (6.13) represents an exactly marginal deformation. Unfortunately however these two terms do not commute, instead they anti-commute due to the presence of the fermion fields $\psi_{i}$. As a result the complete perturbation is not exactly marginal and the $\beta$-function does receive higher order corrections. Thus unlike in the case of section 5, we cannot solve the theory exactly in this case. In particular, we cannot obtain an analytic expression for the boundary state and compute the energy momentum tensor associated with this solution. 
To see this more explicitly we fermionize and rebosonize following the procedure given in refs: [30, 31]. On the boundary the relevant part of these relations take the form:

$$
\begin{gathered}
e^{i Z_{i}(t) / \sqrt{2}}=\frac{1}{\sqrt{2}}\left(\xi^{i}(t)+i \eta^{i}(t)\right) \otimes \tau_{i}, \\
\psi_{i}(t) \eta_{i}(t)=-\frac{i}{\sqrt{2}} \partial \phi_{i}(t),
\end{gathered}
$$

where $\xi_{i}$ and $\eta_{i}$ are Majorana fermions, $\phi_{i}$ are scalars and $\tau_{i}$ are Pauli matrices providing the cocycles needed for the bosonization. In terms of the bosonic field $\phi_{i}$ the perturbation (6.13) can be written as:

$$
\frac{i \widetilde{\lambda}}{4 \sqrt{2}} \int d t\left[\partial_{t} \phi_{1} \otimes \tau_{1} \otimes \sigma_{1}+\partial_{t} \phi_{2} \otimes \tau_{2} \otimes \sigma_{1}\right]
$$

Since $\tau_{1} \otimes \sigma_{1}$ does not commute with $\tau_{2} \otimes \sigma_{1}$, the above perturbation can be thought of as switching on a pair of non-commuting Wilson lines, one along $\phi_{1}$ and the other along $\phi_{2}$, each being of magnitude $\tilde{\lambda} / 4 \sqrt{2}$. As a result the potential does not vanish. Instead we get a contribution proportional to the square of the commutator, which in this case will be equal to $C \widetilde{\lambda}^{4}$ for an approprite positive constant $C$. This gives a contribution to $g(m, \tilde{\lambda})$ equal to $4 C \tilde{\lambda}^{3}$. Eq.(6.5) then gives:

$$
\omega^{2}=\frac{1}{4}-4 C \tilde{\lambda}^{2}
$$

This correction to the value of $\omega$ shows that (6.10) does not represent an exactly marginal deformation.

If we had naively ignored the issue of non-commutativity of the two operators in (6.16) and treated this as an exactly marginal deformation, then we would end up with an expression for the energy momentum tensor similar to that in (5.17), with $f\left(x^{0}\right)$ given by $[4$

$$
f\left(x^{0}\right)=\frac{1}{1+e^{\sqrt{2} x^{0}} \sin ^{2}(\widetilde{\lambda} \pi / 2)}+\frac{1}{1+e^{-\sqrt{2} x^{0}} \sin ^{2}(\widetilde{\lambda} \pi / 2)}-1 .
$$

From this we see that $f\left(\left(x^{0} \pm i y\right) / \sqrt{2}\right)$ has singularities at $x^{0}=\ln \left(1 / \sin ^{2}(\widetilde{\lambda} \pi / 2)\right), y= \pm \pi$, and the energy density blows up at these space-time points. Thus energy flows into these points from the rest of the system. These are precisely the points where we expect the codimension 1 brane-antibrane pairs to form, and the nature of these singularities has precisely the same delta function form as discussed at the end of section 5. Thus one

\footnotetext{
${ }^{16}$ The analysis of $\mathrm{RR}$ charge density shows that $\mathrm{RR}$ charge also accumulates at these points.
} 
might be tempted to interpret this as the creation of brane-antibrane pair. $\square$ However, once we take into account the non-marginality of the perturbation (6.16) this result will be modified. In fact, if the perturbation (6.13) had been exactly marginal, and the energy momentum tensor had been given by (5.17), 6.18), we would have an inconsistency. To see this, note that following arguments similar to those in section 5 we would conclude in this case that the total initial energy of the system is deposited into a codimension 1 brane-antibrane pair, each carrying an energy density equal to:

$$
\frac{1}{\sqrt{2}} \mathcal{T}_{p-1}(1+\cos (\tilde{\lambda} \pi))
$$

where $\mathcal{T}_{p-1}$ is the tension of a BPS D- $(p-1)$ brane. Thus for a generic $\tilde{\lambda}$, (6.19) differs from the tension of a BPS D- $(p-1)$-brane. Unlike in the case of bosonic D-branes, in this case we cannot attribute the excess (deficit) energy to the energy stored in the tachyon field, since there are no tachyons on a BPS D-brane. (The excess energy can still be attributed to other fields on the BPS D-brane, but there is no way a brane can have energy density lower than its tension.) Thus we would be led to an inconsistency, as there will be no interpretation for the final state for $(1+\cos (\pi \tilde{\lambda}))<\sqrt{2}$. Thus it is just as well that (5.17), 6.18) does not give the correct formula for the stress tensor.

\section{Tachyon Dynamics in Closed String Theory}

In this section we briefly discuss possible extensions of the method outlined in this paper to the study of tachyon dynamics in closed string theory. Given a zero momentum tachyon field described by a dimension $(h, h)$ vertex operator $V_{T}$, we try to construct a solution in the Wick rotated theory $\left(x^{0} \rightarrow i x\right)$ by perturbing the orginal CFT by

$$
\tilde{\lambda} \int d^{2} z \cos (\omega X(z, \bar{z})) V_{T}(z, \bar{z})
$$

For $\omega^{2}=4(1-h)$ this would describe a marginal deformation to leading order in $\tilde{\lambda}$. However since in general higher order contribution to the $\beta$-function does not vanish, we do not fix $\omega$ at the beginning. The $\beta$-function associated with the perturbation (7.1) then takes the form:

$$
\beta_{\widetilde{\lambda}}=2\left(\frac{1}{4} \omega^{2}+h-1\right) \tilde{\lambda}+g(\omega, \tilde{\lambda}) .
$$

We now adjust $\omega$ for a given $\tilde{\lambda}$ such that (7.2) vanishes. Thus this perturbation describes a conformal field theory.

\footnotetext{
${ }^{17}$ Production of brane-antibrane pair from the decay of a higher dimensional brane has also been discussed in a different approximation in [5].
} 
The main obstruction to carrying out this program arises from the fact that in this case the operator product of (7.1) with itself does generate other dimension $(1,1)$ operators, - namely the vertex operators associated with the zero momentum graviton and dilaton fields. (The source for the dilaton field manifests itself in the form of a change in the central charge induced by the perturbation.) Thus the rolling tachyon soution acts as a source for the zero momentum graviton and the dilaton fields, and a priori there is no guarantee that we shall be able to solve the equations of motion for these fields perturbatively in

the parameter $\tilde{\lambda}$. (See 32] for an analysis of this problem in non-polynomial closed string field theory [33.) This problem of course is not specific to string theory, and even in the case of a normal scalar field theory coupled to the graviton and the dilaton, there is no guarantee that given a time dependent solution involving the scalar field, we can solve for the graviton and the dilaton field perturbatively in the parameter labelling the solution of the scalar field equations. However, for tachyons localized on a subspace of the full space-time [34], it may be possible to solve the equations of motion of the graviton and the dilaton fields as a perturbation expansion in the deformation parameter.

\section{Conclusion}

In this paper we have discussed a general method for constructing time dependent solutions, describing the rolling of a tachyon on an unstable D-brane system, in cubic open string field theory. We have also provided a description of these solutions as Wick rotated version of euclidean boundary conformal field theories. The family of time dependent solutions associated with different initial conditions correspond to a family of boundary conformal field theories, each of which is related to the original D-brane system by a nearly marginal deformation. The construction can be easily generalized to the case of unstable D-brane systems in superstring theory.

In general these deformed boundary conformal field theories are not exactly solvable, and hence we cannot find explicit analytic expressions for the time dependence of the energy momentum tensor by Wick rotating the boundary state associated with the boundary conformal field theory. However in some special cases the family of boundary CFT's are related by an exactly marginal deformation and are exactly solvable. This allows us to compute the time evolution of the energy momentum tensor explicitly for arbitrary initial condition on the tachyon. One such example discussed in this paper is the case of a bosonic D-p-brane wrapped on a circle of radius $\sqrt{2}$, and we consider the first momentum mode of the tachyon along the circle. Pushing this particular tachyonic mode away from its extremum corresponds to an initial condition where the tachyon field has different signs in different parts of the circle, and hence we might expect that the time evolution of 
the configuration may produce lower dimensional D-branes. We explicitly construct the conserved energy momentum tensor associated with this solution and show that the time evolution of the system does indicate the creation of a codimension 1 brane.

One would like to generalize this to describe creation of a lower dimensional braneantibrane pair due to the decay of an unstable D-brane in superstring theory. But unfortunately the situation here is more complicated as the associated family of conformal field theories are not related to each other by marginal deformation and are not exactly solvable. We hope to return to this problem in the near future.

We conclude with two remarks:

- The solutions constructed here are obtained in classical open string field theory where we ignore coupling to closed string fields. This is expected to be a good approximation in the string coupling $g_{s} \rightarrow 0$ limit. Once we switch on $g_{s}$, two effects will have to be taken into account in the study of the solution: classical backreaction of closed string fields on the solution, and the possibility of the decay into quantum closed string states. The time scale over which these effects will affect the solution is expected to go to infinity in the $g_{s} \rightarrow 0$ limit, but at present we do not know the precise dependence of this time scale on $g_{s}$.

Another parameter that is important in determining the effect of closed string coupling to the solution is $\tilde{\lambda}$, or equivalently, the total energy density of the configuration. We would expect that for small energy density, the effect of coupling to the closed strings will be small, but again we do not know the precise dependence.

- In the study of closed string tachyon condensation[34, renormalization group (RG) flow has played a useful role. The approach taken here is somewhat different: we interpret the complete time dependent solution for a specific initial condition as a single conformal field theory (after Wick rotation). Different inequivalent initial conditions will generate different boundary conformal field theories.

In fact it is possible to argue that at least for open string tachyon condensation, $\mathrm{RG}$ flow can never describe classical time evolution in a strict sense. RG flow takes us from a BCFT with higher boundary entropy to one of lower boundary entropy [21]. In the space-time language this corresponds to interpolating between a D-brane of higher energy to a D-brane of lower energy. It is clear that classical time evolution can never do that; due to conservation of energy it must take us from a D-brane of higher energy to one of lower energy plus other stuff. As a result, the RG analysis can at most give some qualitative information about time evolution in open string theory. 
Acknowledgement: I would like to thank R. Brower, R. Gopakumar, J. Minahan, S. Minwalla, N. Moeller, L. Rastelli and B. Zwiebach for useful discussions, and B. Zwiebach for critical comments on the manuscript. This work was supported in part by a grant from the Eberly College of Science of the Penn State University. I would also like to acknowledge the hospitality of the Center for Theoretical Physics at MIT, and a grant from the NM Rothschild and Sons Ltd at the Isaac Newton Institute where part of this work was done.

\section{References}

[1] G. T. Horowitz and A. R. Steif, Phys. Lett. B 258, 91 (1991); G. Veneziano, arXiv:hep-th/0002094; F. Larsen and F. Wilczek, Phys. Rev. D 55, 4591 (1997) arXiv:hep-th/9610252; G. T. Horowitz and D. Marolf, JHEP 9807, 014 (1998) arXiv:hep-th/9805207; I. I. Kogan and N. B. Reis, Int. J. Mod. Phys. A 16, 4567 (2001) arXiv:hep-th/0107163; J. Khoury, B. A. Ovrut, P. J. Steinhardt and N. Turok, Phys. Rev. D 64, 123522 (2001) arXiv:hep-th/0103239; J. Khoury, B. A. Ovrut, N. Seiberg, P. J. Steinhardt and N. Turok, Phys. Rev. D 65, 086007 (2002) arXiv:hep-th/0108187; P. J. Steinhardt and N. Turok, arXiv:hepth/0111030; N. Seiberg, arXiv:hep-th/0201039; V. Balasubramanian, S. F. Hassan, E. Keski-Vakkuri and A. Naqvi, arXiv:hep-th/0202187; L. Cornalba and M. S. Costa, arXiv:hep-th/0203031; N. A. Nekrasov, arXiv:hep-th/0203112; J. Simon, JHEP 0206, 001 (2002) arXiv:hep-th/0203201; E. Kiritsis and B. Pioline, arXiv:hepth/0204004; H. Liu, G. Moore and N. Seiberg, arXiv:hep-th/0204168; arXiv:hepth/0206182; S. Elitzur, A. Giveon, D. Kutasov and E. Rabinovici, arXiv:hepth/0204189; L. Cornalba, M. S. Costa and C. Kounnas, arXiv:hep-th/0204261; O. Aharony, M. Fabinger, G. T. Horowitz and E. Silverstein, arXiv:hep-th/0204158; B. Craps, D. Kutasov and G. Rajesh, arXiv:hep-th/0205101; S. Kachru, X. Liu, M. B. Schulz and S. P. Trivedi, arXiv:hep-th/0205108; D. Birmingham and M. Rinaldi, arXiv:hep-th/0205246; A. Lawrence, arXiv:hep-th/0205288; V. Balasubramanian and S. F. Ross, arXiv:hep-th/0205290; M. Fabinger and J. McGreevy, arXiv:hepth/0206196; R. Kallosh, L. Kofman, A. D. Linde and A. A. Tseytlin, Phys. Rev. D 64, 123524 (2001) arXiv:hep-th/0106241; G. T. Horowitz and J. Polchinski, arXiv:hepth/0206228; C. M. Chen, D. V. Gal'tsov and M. Gutperle, arXiv:hep-th/0204071; M. Kruczenski, R. C. Myers and A. W. Peet, JHEP 0205, 039 (2002) arXiv:hepth/0204144; S. Roy, arXiv:hep-th/0205198; N. S. Deger and A. Kaya, arXiv:hepth/0206057; J. E. Wang, arXiv:hep-th/0207089. 
[2] M. Gutperle and A. Strominger, arXiv:hep-th/0202210.

[3] A. Sen, arXiv:hep-th/0203211.

[4] A. Sen, arXiv:hep-th/0203265.

[5] K. Hashimoto, arXiv:hep-th/0204203.

[6] S. Sugimoto, S. Terashima arXiv:hep-th/0205085.

[7] J. A. Minahan, arXiv:hep-th/0205098.

[8] A. Ishida and S. Uehara, arXiv:hep-th/0206102.

[9] K. Ohta and T. Yokono, arXiv:hep-th/0207004

[10] K. Bardakci, Nucl. Phys. B 68, 331 (1974) Nucl. Phys. B 70, 397 (1974); Nucl. Phys. B 133, 297 (1978); K. Bardakci and M. B. Halpern, Phys. Rev. D 10, 4230 (1974); Nucl. Phys. B 96, 285 (1975).

[11] O. Bergman and M. R. Gaberdiel, Phys. Lett. B 441, 133 (1998) arXiv:hepth/9806155; Class. Quant. Grav. 17, 961 (2000) arXiv:hep-th/9908126].

[12] A. Sen, JHEP 9810, 021 (1998) arXiv:hep-th/9809111.

[13] G. W. Gibbons, arXiv:hep-th/0204008; M. Fairbairn and M. H. Tytgat, arXiv:hepth/0204070; S. Mukohyama, arXiv:hep-th/0204084; A. Feinstein, arXiv:hepth/0204140; T. Padmanabhan, arXiv:hep-th/0204150; A. Frolov, L. Kofman and A. A. Starobinsky, arXiv:hep-th/0204187; D. Choudhury, D. Ghoshal, D. P. Jatkar and S. Panda, arXiv:hep-th/0204204; X. Li, J. Hao and D. Liu, arXiv:hepth/0204252; G. Shiu and I. Wasserman, arXiv:hep-th/0205003; T. Padmanabhan and T. R. Choudhury, arXiv:hep-th/0205055; L. Kofman and A. Linde, arXiv:hepth/0205121; M. Sami, arXiv:hep-th/0205146; M. Sami, P. Chingangbam and T. Qureshi, arXiv:hep-th/0205179; T. Mehen and B. Wecht, arXiv:hep-th/0206212.

[14] G. R. Dvali and S. H. Tye, Phys. Lett. B 450, 72 (1999) arXiv:hep-ph/9812483; C. Acatrinei and C. Sochichiu, arXiv:hep-th/0104263; S. H. Alexander, Phys. Rev. D 65, 023507 (2002) arXiv:hep-th/0105032; G. R. Dvali, Q. Shafi and S. Solganik, arXiv:hep-th/0105203; C. P. Burgess, M. Majumdar, D. Nolte, F. Quevedo, G. Rajesh and R. Z. Zhang, JHEP 0107, 047 (2001) arXiv:hep-th/0105204; A. Mazumdar, S. Panda and A. Perez-Lorenzana, Nucl. Phys. B 614, 101 (2001) arXiv:hepph/0107058; C. P. Burgess, P. Martineau, F. Quevedo, G. Rajesh, and R. J. Zhang, 
JHEP 0203, 052 (2002) arXiv:hep-th/0111025; S. Sarangi and S. H. Tye, arXiv:hepth/0204074; S. Corley and D. A. Lowe, Phys. Lett. B 528, 139 (2002) arXiv:hepth/0108178].

[15] E. Gava, K. S. Narain and M. H. Sarmadi, Nucl. Phys. B 504, 214 (1997) arXiv:hepth/9704006].

[16] A. Hashimoto and W. I. Taylor, Nucl. Phys. B 503, 193 (1997) arXiv:hepth/9703217.

[17] J. Garcia-Bellido, R. Rabadan and F. Zamora, JHEP 0201, 036 (2002) arXiv:hepth/0112147; R. Blumenhagen, B. Kors, D. Lust and T. Ott, arXiv:hep-th/0202124; K. Dasgupta, C. Herdeiro, S. Hirano and R. Kallosh, Phys. Rev. D 65, 126002 (2002) arXiv:hep-th/0203019; N. Jones, H. Stoica and S. H. Tye, arXiv:hep-th/0203163.

[18] N. Moeller and B. Zwiebach, arXiv:hep-th/0207107.

[19] H. Goldstein, Classical Mechanics, Addison Wesley.

[20] A. B. Zamolodchikov, Sov. J. Nucl. Phys. 46, 1090 (1987) [Yad. Fiz. 46, 1819 (1987)].

[21] I. Affleck and A. W. Ludwig, Phys. Rev. Lett. 67, 161 (1991); P. Fendley, H. Saleur and N. P. Warner, Nucl. Phys. B 430, 577 (1994) arXiv:hep-th/9406125; J. A. Harvey, D. Kutasov and E. J. Martinec, arXiv:hep-th/0003101.

[22] A. A. Gerasimov and S. L. Shatashvili, JHEP 0010, 034 (2000) arXiv:hepth/0009103]; D. Kutasov, M. Marino and G. W. Moore, JHEP 0010, 045 (2000) arXiv:hep-th/0009148; arXiv:hep-th/0010108; D. Ghoshal and A. Sen, JHEP 0011, 021 (2000) arXiv:hep-th/0009191.

[23] S. Dasgupta and T. Dasgupta, JHEP 0106, 007 (2001) arXiv:hep-th/0010247.

[24] J. Polchinski and Y. Cai, Nucl. Phys. B 296, 91 (1988); C. G. Callan, C. Lovelace, C. R. Nappi and S. A. Yost, Nucl. Phys. B 460, 351 (1996) arXiv:hep-th/9510161; M. Li, Nucl. Phys. B 460, 351 (1996) arXiv:hep-th/9510161]; O. Bergman and M. R. Gaberdiel, Nucl. Phys. B 499, 183 (1997) arXiv:hep-th/9701137.

[25] M. B. Green and M. Gutperle, Nucl. Phys. B 476, 484 (1996) [arXiv:hep-th/9604091; P. Di Vecchia, M. Frau, I. Pesando, S. Sciuto, A. Lerda and R. Russo, Nucl. Phys. B 507, 259 (1997) arXiv:hep-th/9707068; P. Di Vecchia and A. Liccardo, arXiv:hepth/9912275. 
[26] C. G. Callan and I. R. Klebanov, Phys. Rev. Lett. 72, 1968 (1994) arXiv:hepth/9311092]; C.G. Callan, I.R. Klebanov, A.W. Ludwig and J.M. Maldacena, Nucl. Phys. B422, 417 (1994) hep-th/9402113; J. Polchinski and L. Thorlacius, Phys. Rev. D50, 622 (1994) hep-th/9404008.

[27] A. Recknagel and V. Schomerus, Nucl. Phys. B545, 233 (1999) hep-th/9811237.

[28] M. R. Gaberdiel and A. Recknagel, JHEP 0111, 016 (2001) arXiv:hep-th/0108238.

[29] D. Friedan, E. J. Martinec and S. H. Shenker, Nucl. Phys. B 271, 93 (1986).

[30] A. Sen, JHEP 9809, 023 (1998) [hep-th/9808141; JHEP 9812, 021 (1998) arXiv:hep-th/9812031.

[31] J. Majumder and A. Sen, JHEP 0006, 010 (2000) arXiv:hep-th/0003124.

[32] S. Mukherji and A. Sen, Nucl. Phys. B 363, 639 (1991).

[33] B. Zwiebach, Nucl. Phys. B 390, 33 (1993) arXiv:hep-th/9206084; arXiv:hepth/9305026; Annals Phys. 267, 193 (1998) [arXiv:hep-th/9705241].

[34] A. Adams, J. Polchinski and E. Silverstein, JHEP 0110, 029 (2001) arXiv:hepth/0108075; A. Dabholkar, arXiv:hep-th/0109019; J. G. Russo and A. A. Tseytlin, JHEP 0111, 065 (2001) arXiv:hep-th/0110107; A. Dabholkar, Phys. Rev. Lett. 88, 091301 (2002) arXiv:hep-th/0111004; C. Vafa, arXiv:hep-th/0111051; J. A. Harvey, D. Kutasov, E. J. Martinec and G. Moore, arXiv:hep-th/0111154; A. Dabholkar and C. Vafa, JHEP 0202, 008 (2002) arXiv:hep-th/0111155]; Y. Michishita and P. Yi, Phys. Rev. D 65, 086006 (2002) arXiv:hep-th/0111199]; J. R. David, M. Gutperle, M. Headrick and S. Minwalla, JHEP 0202, 041 (2002) arXiv:hep-th/0111212; S. k. Nam and S. J. Sin, arXiv:hep-th/0201132; S. P. De Alwis and A. T. Flournoy, arXiv:hep-th/0201185; S. J. Sin, arXiv:hep-th/0202097; R. Rabadan and J. Simon, tachyons," JHEP 0205, 045 (2002) arXiv:hep-th/0203243; A. Basu, arXiv:hepth/0204247. 